\title{
BLOCKS AND CUT VERTICES OF THE BUNEMAN GRAPH
}

\author{
A. W. M. DRESS, K. T. HUBER, J. KOOLEN, V. MOULTON
}

\begin{abstract}
Given a set $\Sigma$ of bipartitions of some finite set $X$ of cardinality at least 2 , one can associate to $\Sigma$ a canonical $X$-labeled graph $\mathcal{B}(\Sigma)$, called the Buneman graph. This graph has several interesting mathematical properties - for example, it is a median network and therefore an isometric subgraph of a hypercube. It is commonly used as a tool in studies of DNA sequences gathered from populations. In this paper, we present some results concerning the cut vertices of $\mathcal{B}(\Sigma)$, i.e., vertices whose removal disconnect the graph, as well as its blocks or 2-connected components - results that yield, in particular, an intriguing generalization of the wellknown fact that $\mathcal{B}(\Sigma)$ is a tree if and only if any two splits in $\Sigma$ are compatible.
\end{abstract}

Keywords: Split, split system, Buneman graph, median graph, cut vertex, block, compatible partitions, $X$-tree, phylogenetics

Classification numbers: 05C05, 05C40, 05C90, 92B10, 92D15

\section{INTRODUCTION}

Consider a finite set $X$ of cardinality at least 2 . We denote by $\bar{A}$ the complement $X-A$ for any subset $A$ of $X$. And we call a bipartition $S=$ $\{A, B\}$ into a proper non-empty subset $A$ of $X$ and its complement $B=$ $\bar{A}$ a split or, more specifically, an $X$-split. For any non-empty collection $\Sigma$ of $X$-splits, we define the Buneman graph $\mathcal{B}(\Sigma)=(V(\Sigma), E(\Sigma))$ to be the graph whose vertex set $V(\Sigma)$ consists of all maps $\phi$ from $\Sigma$ into the power set $\mathcal{P}(X)$ of $X$ that satisfy, for all $S, S^{\prime} \in \Sigma$, the following two conditions

(BG1) $\phi(S) \in S$, i.e., if $S=\{A, B\}$, then $\phi(S)=A$ or $\phi(S)=B$, and

(BG2) $\phi(S) \cap \phi\left(S^{\prime}\right) \neq \emptyset$.

And we define its edge set $E(\Sigma)$ to consist of all those subsets $\{\phi, \psi\}$ of $V(\Sigma)$ for which the difference set $\Delta(\phi, \psi)$, defined by

$$
\Delta(\phi, \psi):=\{S \in \Sigma: \phi(S) \neq \psi(S)\},
$$

has cardinality 1.

We also denote by $V^{*}(\Sigma)$ the superset of $V(\Sigma)$ consisting of all maps $\phi: \Sigma \rightarrow \mathcal{P}(X)$ that just satisfy (BG1) (but not necessarily (BG2)). And we define the extended Buneman graph $\mathcal{B}^{*}(\Sigma)=\left(V^{*}(\Sigma), E^{*}(\Sigma)\right)$

Date: 19.02 .2010$. 
to be the (necessarily connected) graph with vertex set $V^{*}(\Sigma)$ and edge set $E^{*}(\Sigma)$ defined exactly as $E(\Sigma)$ above, yet with $V(\Sigma)$ replaced by $V^{*}(\Sigma)$ in its definition. Note that $\mathcal{B}^{*}(\Sigma)$ is clearly isomorphic to an $|\Sigma|$-dimensional hypercube.

An example of a Buneman graph is pictured in Figure 1. Note that some of the vertices of the graph are labelled by elements in $X$. This arises from a canonical labelling map $\varphi_{\Sigma}: X \rightarrow V(\Sigma): x \mapsto \phi_{x}$ mapping $X$ into the vertex set of $\mathcal{B}(\Sigma)$, where, for each $x \in X, \phi_{x}$ denotes the map in $V(\Sigma)$ that associates, to any $S \in \Sigma$, that subset $S(x)$ of $X$ in $S$ that contains $x$. Clearly, this map is necessarily contained in $V(\Sigma)$. In particular, the cardinality of $V(\Sigma)$ must be at least 2 for any non-empty collection $\Sigma$ of $X$-splits.

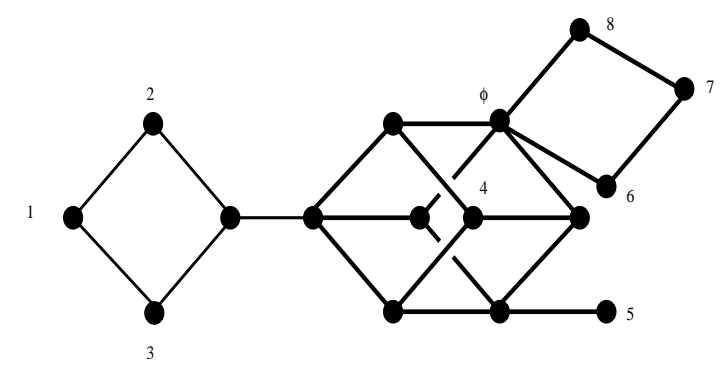

Figure 1. For $X:=\{1,2, \ldots, 8\}$, the Buneman graph $\mathcal{B}\left(\Sigma_{8}\right)$ for the collection $\Sigma_{8}$ of $X$-splits given by $\Sigma_{8}:=\left\{S_{13}, S_{12}, S_{123}, S_{1235}, S_{45}, S_{1234}, S_{67}, S_{78}, S_{5}\right\}$ (where, for example, $S_{13}$ denotes the $X$-split $\{\{1,3\},\{2,4,5,6,7,8\}\})$. The vertex $\phi$ is a cut-vertex of $\mathcal{B}\left(\Sigma_{8}\right)$.

The Buneman graph has appeared in the literature in various guises: As a co-pair hypergraph in [1, 2], as a special type of median graph in e.g. [3] (see also [20] for a review of median graphs), and in the above form in [12]. In addition, Buneman graphs and median networks are regularly used to help analyze viral or mitochondrial sequence data gathered from populations (see e.g. [5, 10]).

It is a well-known basic fact essentially established by Peter Buneman in 1971 (cf. [8]) that $\mathcal{B}(\Sigma)$ is a tree if and only if any two splits $S, S^{\prime}$ in $\Sigma$ are compatible, i.e., if and only if, for any two splits $S=\{A, B\}, S^{\prime}=$ $\left\{A^{\prime}, B^{\prime}\right\}$ in $\Sigma$, one of the four intersections $A \cap A^{\prime}, A \cap B^{\prime}, B \cap A^{\prime}, B \cap B^{\prime}$ is empty.

Here, we will generalize this fact in a rather natural way as follows: Given any split system $\Sigma$, let $\pi_{0}(\Sigma)$ denote the set of connected components of the so-called incompatibility graph $\Gamma(\Sigma)$ of $\Sigma$, i.e., the graph with vertex set $\Sigma$ whose edge set consists of all pairs of splits contained in $\Sigma$ that are not compatible. For example, for $\Sigma_{8}$ the collection of splits in Figure 1, $\Gamma\left(\Sigma_{8}\right)$ consists of five cliques, viz $\left\{S_{13}, S_{12}\right\},\left\{S_{123}\right\}$, $\left\{S_{1234}, S_{1235}, S_{45}\right\},\left\{S_{67}, S_{78}\right\}$, and $\left\{S_{5}\right\}$. 
Further, let $\mathcal{T}(\Sigma)$ denote the (by construction necessarily bipartite) graph with vertex set $\mathcal{V}(\Sigma)$ the disjoint union of $V(\Sigma)$ and $\pi_{0}(\Sigma)$ and edge set $\mathcal{E}(\Sigma)$ the set of all pairs $\left\{\Sigma_{0}, \phi\right\}$ with $\Sigma_{0} \in \pi_{0}(\Sigma), \phi \in V(\Sigma)$, and $\phi(S) \cap A_{0} \neq \emptyset$ for all $S \in \Sigma-\Sigma_{0}$ and all $A_{0} \in S_{0}$ for some or, equivalently, every - split $S_{0} \in \Sigma_{0}$. Then, this graph $\mathcal{T}(\Sigma)=$ $(\mathcal{V}(\Sigma), \mathcal{E}(\Sigma)$ ) is always a tree (Theorem 6.1). For example, the tree $\mathcal{T}\left(\Sigma_{8}\right)$ for the split system $\Sigma_{8}$ referred to in Figure 1 is presented in Figure 2 (a) in the last section.

To establish this fact, we will first introduce appropriate notations and discuss some generalities in Section 2, Then, in the next section, we will study the cut vertices of $\mathcal{B}(\Sigma)$, that is, the vertices $\phi \in V(\Sigma)$ of $\mathcal{B}(\Sigma)$ for which the induced subgraph $\mathcal{B}^{(\phi)}(\Sigma)$ of $\mathcal{B}(\Sigma)$ with vertex set $V(\Sigma)-\{\phi\}$ is disconnected (see e.g. Figure 1). Remarkably, these vertices can be characterized in quite a few equivalent ways (cf. Theorem 4.1). In particular, given any map $\phi \in V(\Sigma)$, one can define as many as at least eight significantly distinct graphs all of which have the property that there is a canonical one-to-one correspondence between their connected components and the connected components of $\mathcal{B}^{(\phi)}(\Sigma)$. So, a map $\phi \in V(\Sigma)$ is a cut vertex if and only if any of these graphs is disconnected.

In Section 5, we shall use the cut vertices to provide an explicit description of the blocks (or two-connected components) of $\mathcal{B}(\Sigma)$, , that is, the maximal subsets $V$ of $V(\Sigma)$ for which the graph induced on $V$ is connected and does not contain a cut vertex. More specifically, we will show that there is a canonical one-to-one correspondence between $\pi_{0}(\Sigma)$ and the set $B l(\Sigma)$ of blocks of $\mathcal{B}(\Sigma)$ given by associating, to each connected component $\Sigma_{0} \in \pi_{0}(\Sigma)$ of $\Gamma(\Sigma)$, the set $B\left(\Sigma_{0}\right)$ consisting of all maps $\phi \in V(\Sigma)$ with $\left\{\Sigma_{0}, \phi\right\} \in \mathcal{E}(\Sigma)$, see Theorem 5.1 for details. Thus, for example, we see that in Figure 1 the Buneman graph has 5 blocks that correspond to the 5 cliques in the associated incompatibility graph.

And finally, in the last section, we will establish the above-mentioned generalization of Peter Buneman's result from 1971. In addition, we will establish some refinements that will allow us to associate, to any split system $\Sigma$, a "proper" $X$-tree $T_{\Sigma}$, i.e., a triple $\left(V_{\Sigma}, E_{\Sigma} ; \Phi_{\Sigma}\right)$ consisting of a tree with vertex set $V_{\Sigma}$ and edge set $E_{\Sigma}$, and a labelling map $\Phi_{\Sigma}: X \rightarrow V_{\Sigma}$ such that the degree of any vertex in $V_{\Sigma}$ that is not contained in the image of $\Phi_{\Sigma}$ is at least 3 . For example, the tree $T_{\Sigma_{8}}$ for the split system $\Sigma_{8}$ in Figure 1 is presented in Figure 2 (b).

Note that besides providing important structural information concerning the Buneman graph (and median networks — see e.g. [7]), cut vertices have been used to help in the computation of most parsimonious trees for DNA-sequence data (see e.g. [4, 6, 222]). And the blocks of $\mathcal{B}(\Sigma)$ determined by them are also closely related to the "blobs" defined in terms of directed graphs described by D. Gusfield et al. in [18] (see also e.g. [17, 19]). 
In [15], we will use the results described above to show that $\mathcal{B}(\Sigma)$ can be described as a push-out in terms of the data involved in the construction of $\mathcal{T}(\Sigma)$, and discuss further potential applications.

\section{PRELIMINARIES}

In this section, we will review some results concerning the Buneman graph that will be needed later on. Wherever appropriate, we will refer the reader to the relevant literature for proofs of the results that we state. We also refer the reader to [9] for the basic terminology of graph theory that we will use throughout this paper.

First, we define, for every map $\phi \in V^{*}(\Sigma)$ and every subset $\Xi$ of $\Sigma$, the map $\phi^{\Xi} \in V^{*}(\Sigma)$ by putting

$$
\phi^{\Xi}(S):=\left\{\begin{array}{l}
\overline{\phi(S)} \text { if } S \in \Xi, \\
\phi(S) \text { else, }
\end{array}\right.
$$

for every split $S \in \Sigma$. Note that, writing $\phi^{S}$ rather than $\phi^{\Xi}$ in case $\Xi$ consists of a single split $S$, only, one has $\{\phi, \psi\} \in E^{*}(\Sigma)$ for some $\phi, \psi \in V^{*}(\Sigma)$ if and only if $\psi=\phi^{S}$ holds for some (necessarily unique) split $S \in \Sigma$, viz the unique split $S=S_{\phi, \psi}$ in $\Delta(\phi, \psi)$. Note also that, for a fixed map $\phi \in V^{*}(\Sigma)$ and any other map $\psi \in V^{*}(\Sigma)$, one clearly has $\phi^{\Delta(\phi, \psi)}=\psi$. In particular, $\psi$ is completely determined by $\phi$ and the difference set $\Delta(\phi, \psi)$.

Using other notations and arguments, the following result has also (at least implicitly) been shown in, e.g., [21, Chapter 3.8] and [2, Chapter 5.1]. It is also related to [18, Theorem 1].

Lemma 2.1. Given a vertex $\phi$ in $V(\Sigma)$ and a split $S \in \Sigma$, the following three assertions are equivalent:

(i) $\phi(S)$ is contained in the set $\min (\phi[\Sigma])$ of inclusion-minimal subsets in the image $\phi[\Sigma]$ of $\Sigma$ relative to $\phi$,

(ii) the map $\phi^{S} \in V^{*}(\Sigma)$ is, in fact, a vertex in $V(\Sigma)$ and thus forms, together with $\phi$, an edge in $E(\Sigma)$,

(iii) there exists some vertex $\psi$ in $V(\Sigma)$ with $\phi(S) \in \min (\phi[\Delta(\phi, \psi)])$.

Proof: (i) $\Rightarrow$ (ii): Suppose $S \in \Sigma$ with $\phi(S) \in \min (\phi[\Sigma])$. To see that $\phi^{S} \in V(\Sigma)$ holds, note that, by definition, we have $\phi^{S}\left(S_{1}\right) \cap \phi^{S}\left(S_{2}\right)=$ $\phi\left(S_{1}\right) \cap \phi\left(S_{2}\right) \neq \emptyset$ for all $S_{1}, S_{2} \in \Sigma$ that are distinct from $S$. Moreover, we have $\phi^{S}\left(S^{\prime}\right) \cap \phi^{S}(S)=\phi\left(S^{\prime}\right) \cap \overline{\phi(S)} \neq \emptyset$ for all $S^{\prime} \in \Sigma-\{S\}$ as $\phi\left(S^{\prime}\right) \cap \overline{\phi(S)}=\emptyset$ would imply $\phi\left(S^{\prime}\right) \subset \phi(S)$ in contradiction to $\phi(S) \in \min (\phi[\Sigma])$.

(ii) $\Rightarrow$ (iii): This is trivial: Just put $\psi:=\phi^{S}$.

(iii) $\Rightarrow$ (i): This follows immediately from the following, slightly more general observation:

Lemma 2.2. Given any two vertices $\phi, \psi$ in $\mathcal{B}(\Sigma)$, the set $\phi[\Delta(\phi, \psi)]$ is an ideal in the partially ordered set $\phi[\Sigma]$, that is, $A_{1}, A_{2} \in \phi[\Sigma]$, $A_{1} \in \phi[\Delta(\phi, \psi)]$, and $A_{2} \subseteq A_{1}$ implies $A_{2} \in \phi[\Delta(\phi, \psi)]$. 
Proof: Indeed, denoting by $S_{1}$ and $S_{2}$ the two splits in $\Sigma$ with $\phi\left(S_{1}\right)=$ $A_{1}$ and $\phi\left(S_{2}\right)=A_{2}$, respectively, we must have $S_{1} \in \Delta(\phi, \psi)$. Thus, our assumption $A_{2} \subseteq A_{1}=\phi\left(S_{1}\right)$ implies $\emptyset=A_{2} \cap \overline{\phi\left(S_{1}\right)}=A_{2} \cap \psi\left(S_{1}\right)$ and, hence, $\psi\left(S_{2}\right) \neq A_{2}=\phi\left(S_{2}\right)$, i.e., $S_{2} \in \Delta(\phi, \psi)$, as claimed.

This finishes also the proof of Lemma 2.1.

Next, note that, by definition, $\mathcal{B}(\Sigma)$ is clearly the induced subgraph of $\mathcal{B}^{*}(\Sigma)$ with vertex set $V(\Sigma)$, and the graph-theoretical distance $D^{*}\left(\psi, \psi^{\prime}\right)$ between any two vertices $\psi$ and $\psi^{\prime}$ in $\mathcal{B}^{*}(\Sigma)$ coincides with the cardinality of their difference set $\Delta\left(\psi, \psi^{\prime}\right)$.

It follows that $\mathcal{B}^{*}(\Sigma)$ is, in particular, a median graph. I.e., there exists, for any three maps $\phi_{1}, \phi_{2}, \phi_{3} \in V^{*}(\Sigma)$, a unique map med $\left(\phi_{1}, \phi_{2}, \phi_{3}\right)$ in $V^{*}(\Sigma)$, dubbed the median of $\phi_{1}, \phi_{2}$, and $\phi_{3}$, that lies simultaneously on (i) a shortest path joining $\phi_{1}$ to $\phi_{2}$, (ii) a shortest path joining $\phi_{1}$ to $\phi_{3}$, and (iii) a shortest path joining $\phi_{2}$ to $\phi_{3}$. It maps every split $S \in \Sigma$ onto

$$
\operatorname{med}\left(\phi_{1}, \phi_{2}, \phi_{3}\right)(S):= \begin{cases}\phi_{1}(S) & \text { if } \phi_{1}(S) \in\left\{\phi_{2}(S), \phi_{3}(S)\right\} \\ \phi_{2}(S) & \text { otherwise }\end{cases}
$$

Parts of the following corollary have also been observed in, e.g., [1, Section 2.1], [1, Proposition 1], [3], and [21]:

Corollary 2.3. The Buneman graph $\mathcal{B}(\Sigma)$ is, for every split system $\Sigma$, a connected isometric and median subgraph of the extended Buneman graph $\mathcal{B}^{*}(\Sigma)$ associated with $\Sigma$. That is:

- The median med $\left(\phi_{1}, \phi_{2}, \phi_{3}\right)$ of any three maps $\phi_{1}, \phi_{2}, \phi_{3} \in V(\Sigma)$ is also contained in $V(\Sigma)$.

- The graph-theoretical distance $D\left(\psi, \psi^{\prime}\right)$ between any two vertices $\psi$ and $\psi^{\prime}$ in $\mathcal{B}(\Sigma)$ is finite and coincides with the distance $D^{*}\left(\psi, \psi^{\prime}\right)$ between $\psi$ and $\psi^{\prime}$ in the larger graph $\mathcal{B}^{*}(\Sigma)$ : I.e., there exists, for any two vertices $\psi, \psi^{\prime} \in V(\Sigma)$, a sequence $\psi_{0}:=\psi, \psi_{1}, \ldots, \psi_{k}:=\psi^{\prime}$ of maps in $V(\Sigma)$ of length $k:=\left|\Delta\left(\psi, \psi^{\prime}\right)\right|$ such that $\left|\Delta\left(\psi_{i-1}, \psi_{i}\right)\right|=1$ holds for all $i=1, \ldots, k$.

More specifically, there exists a canonical one-to-one correspondence between the set consisting of all such sequences $\psi_{0}=\psi, \psi_{1}, \ldots, \psi_{\left|\Delta\left(\psi, \psi^{\prime}\right)\right|}=$ $\psi^{\prime}$ and the set of all linear orders "․ defined on $\psi\left[\Delta\left(\psi, \psi^{\prime}\right)\right]$ that extend the partial order of $\psi\left[\Delta\left(\psi, \psi^{\prime}\right)\right]$ defined by set inclusion.

Furthermore, the following holds:

(i) Given any three vertices $\psi, \psi^{\prime}, \phi \in V(\Sigma)$, every shortest path $\psi_{0}:=\psi, \psi_{1}, \ldots, \psi_{\left|\Delta\left(\psi, \psi^{\prime}\right)\right|}:=\psi^{\prime}$ connecting $\psi$ and $\psi^{\prime}$ in $\mathcal{B}(\Sigma)$ must pass through $\phi$ if and only if one has $\Delta\left(\psi, \psi^{\prime}\right)=\Delta(\psi, \phi) \cup$ $\Delta\left(\phi, \psi^{\prime}\right)$ and $\psi(S) \subset \psi\left(S^{\prime}\right)$ for every $S \in \Delta(\psi, \phi)$ and $S^{\prime} \in$ $\Delta\left(\phi, \psi^{\prime}\right)$. And, conversely, there exists a shortest path $\psi_{0}:=$ $\psi, \psi_{1}, \ldots, \psi_{\left|\Delta\left(\psi, \psi^{\prime}\right)\right|}:=\psi^{\prime}$ connecting $\psi$ and $\psi^{\prime}$ in $\mathcal{B}(\Sigma)$ not passing through $\phi$ if and only if there exist splits $S \in \Delta(\psi, \phi)$ and $S^{\prime} \in \Delta\left(\psi^{\prime}, \phi\right)$ with $\phi(S) \cup \phi\left(S^{\prime}\right) \neq X$. 
(ii) Any cycle $\left\{\psi_{0}, \psi_{1}\right\},\left\{\psi_{1}, \psi_{2}\right\}, \ldots,\left\{\psi_{n-1}, \psi_{n}:=\psi_{0}\right\}$ in $E(\Sigma)$ that is not the "mod-2 sum" - or symmetric difference - of cycles of smaller length, is of length 4 (as this is easily seen to hold for any median graph).

Proof: As, in view of (1), there exists, for all $\phi_{1}, \phi_{2}, \phi_{3} \in V^{*}(\Sigma)$ and all $S, S^{\prime} \in \Sigma$, an index $i \in\{1,2,3\}$ with $\operatorname{med}\left(\phi_{1}, \phi_{2}, \phi_{3}\right)(S)=\phi_{i}(S)$ and $\operatorname{med}\left(\phi_{1}, \phi_{2}, \phi_{3}\right)\left(S^{\prime}\right)=\phi_{i}\left(S^{\prime}\right)$, it is obvious that $\operatorname{med}\left(\phi_{1}, \phi_{2}, \phi_{3}\right) \in V(\Sigma)$ holds for any three maps $\phi_{1}, \phi_{2}, \phi_{3} \in V(\Sigma)$.

Further, with $\psi, \psi^{\prime} \in V(\Sigma)$ as above, select a split $S \in \Delta\left(\psi, \psi^{\prime}\right)$ such that $\psi(S) \in \min \psi\left[\Delta\left(\psi, \psi^{\prime}\right)\right]$ holds, and put $\psi_{1}:=\psi^{S}$. Clearly, $\psi_{1} \in$ $V(\Sigma),\left\{\psi, \psi_{1}\right\} \in E(\Sigma), \Delta\left(\psi, \psi_{1}\right)=\{S\}$, and $\Delta\left(\psi_{1}, \psi^{\prime}\right)=\Delta\left(\psi, \psi^{\prime}\right)-$ $\{S\}$ and, therefore, $\psi_{1}\left[\Delta\left(\psi_{1}, \psi^{\prime}\right)\right]=\psi\left[\Delta\left(\psi, \psi^{\prime}\right)\right]-\{\psi(S)\}$ also holds. Thus, in view of Lemma 2.1, the first assertion in the statement of the corollary follows easily using induction with respect to $\left|\Delta\left(\psi, \psi^{\prime}\right)\right|$.

The remaining assertions now follow from this assertion: It implies that, given any three vertices $\psi, \psi^{\prime}, \phi \in V(\Sigma)$, the following two claims are equivalent:

(i) Every shortest path $\psi_{0}:=\psi, \psi_{1}, \ldots, \psi_{\left|\Delta\left(\psi, \psi^{\prime}\right)\right|}:=\psi^{\prime}$ connecting $\psi$ and $\psi^{\prime}$ must pass through $\phi$.

(ii) Both $\Delta\left(\psi, \psi^{\prime}\right)=\Delta(\phi, \psi) \cup \Delta\left(\phi, \psi^{\prime}\right)$ and $\psi(S) \preceq \psi\left(S^{\prime}\right)$ hold, for all $S \in \Delta(\phi, \psi)$ and $S^{\prime} \in \Delta\left(\phi, \psi^{\prime}\right)$, for every linear order " $\preceq$ " defined on $\psi\left[\Delta\left(\psi, \psi^{\prime}\right)\right]$ that extends the partial order of $\psi[\Delta(\phi, \psi)]$ defined by set inclusion.

Next, we associate to every edge $e=\{\phi, \psi\} \in E^{*}(\Sigma)$ its type $\kappa(e)$ that we define to be the unique split $\kappa(e):=S_{\phi, \psi}$ in $\Delta(\phi, \psi)$. This clearly yields a surjective map $\kappa: E^{*}(\Sigma) \rightarrow \Sigma$ whose restriction to $E(\Sigma)$ we denote by $\kappa_{\Sigma}$.

For example, the 4 horizontal edges of the small cube in the middle of Figure 1 all are of type $S_{1234}$, and the 4 parallel edges in that cube going from SW to NE are all of type $S_{1235}$.

Clearly, one has $\Delta(\phi, \psi)=\left\{\kappa\left(\left\{\phi_{i-1}, \phi_{i}\right\}\right): i=1, \ldots,|\Delta(\phi, \psi)|\right\}$ for every shortest path $\phi_{0}:=\phi, \phi_{1}, \ldots, \phi_{|\Delta(\phi, \psi)|}:=\psi$ from a vertex $\phi$ to a vertex $\psi$ in $\mathcal{B}(\Sigma)$ or $\mathcal{B}^{*}(\Sigma)$. We claim (see also [21, Exercise 11, p.64])

Lemma 2.4. The set $\kappa_{\Sigma}^{-1}(S)$ is, for every split $S=\{A, B\} \in \Sigma$, a cutset of $\mathcal{B}(\Sigma)$ "inducing" the split $S$. That is, removing the edges in $\kappa_{\Sigma}^{-1}(S)$ from $\mathcal{B}(\Sigma)$ yields a subgraph with precisely two connected components, one denoted by $\mathcal{B}(\Sigma \mid A)$ containing all the vertices $\phi_{a}$ with $a \in A$, and the other one denoted by $\mathcal{B}(\Sigma \mid B)$ containing all the vertices $\phi_{b}$ with $b \in B$. Furthermore, the same holds (mutatis mutandi) for $\mathcal{B}^{*}(\Sigma)$ and the subset $\kappa^{-1}(S)$ of $E^{*}(\Sigma)$.

Proof: Let $S=\{A, B\}$ be an arbitrary split in $\Sigma$. It suffices to show that the two subsets $\mathcal{B}(\Sigma \mid A):=\{\phi \in V(\Sigma): \phi(S)=A\} \supseteq\left\{\phi_{a}: a \in A\right\}$ and $\mathcal{B}(\Sigma \mid B):=\{\phi \in V(\Sigma): \phi(S)=B\} \supseteq\left\{\phi_{b}: b \in B\right\}$ of $V(\Sigma)$ are 
(exactly the two) connected components of the subgraph of $\mathcal{B}(\Sigma)$ obtained by removing the edges in $\kappa_{\Sigma}^{-1}(S)$. Indeed, it follows immediately from the definition of $E(\Sigma)$ that every path in $\mathcal{B}(\Sigma)$ from a vertex in $\mathcal{B}(\Sigma \mid A)$ to a vertex in $\mathcal{B}(\Sigma \mid B)$ must contain at least one edge in $\kappa_{\Sigma}^{-1}(S)$. Moreover, in view of Corollary 2.3, no shortest path between any two vertices in $\mathcal{B}(\Sigma \mid A)$ (and, analogously, in $\mathcal{B}(\Sigma \mid B)$ ), can pass through an edge in $\kappa^{-1}(S)$. And, clearly, the same argument works just as well for $\mathcal{B}^{*}(\Sigma)$.

Note that restricting the maps $\phi$ in $V(\Sigma)$ to a given subset $\Sigma^{\prime}$ of $\Sigma$ clearly induces a surjective graph morphism from $\mathcal{B}^{*}(\Sigma)$ to $\mathcal{B}^{*}\left(\Sigma^{\prime}\right)$. That is, it yields a surjective map from $V^{*}(\Sigma)$ onto $V^{*}\left(\Sigma^{\prime}\right)$ that maps every edge $e$ in $E^{*}(\Sigma)$ either onto a single vertex (if $\kappa(e) \in \Sigma-\Sigma^{\prime}$ ) in $V^{*}\left(\Sigma^{\prime}\right)$ or onto an edge in $E^{*}\left(\Sigma^{\prime}\right)$ (if $\left.\kappa(e) \in \Sigma^{\prime}\right)$. We now show that it also induces a surjective graph morphism $\operatorname{res}_{\Sigma \rightarrow \Sigma^{\prime}}$ from $\mathcal{B}(\Sigma)$ onto $\mathcal{B}\left(\Sigma^{\prime}\right)$ (see also [12]):

Lemma 2.5. Given a subset $\Sigma^{\prime}$ of a split system $\Sigma$ on $X$, and a map $\psi \in V\left(\Sigma^{\prime}\right)$, there exists (at least) one extension $\phi$ of $\psi$ in $V(\Sigma)$, i.e., a $\operatorname{map} \phi \in V(\Sigma)$ with $\left.\phi\right|_{\Sigma^{\prime}}=\psi$.

Moreover, the resulting surjective graph morphism res $_{\Sigma \rightarrow \Sigma^{\prime}}$ contracts every edge $e \in E(\Sigma)$ that is of type $\kappa(e) \in \Sigma-\Sigma^{\prime}$ onto a vertex while it maps every edge $e \in E(\Sigma)$ of type $\kappa(e) \in \Sigma^{\prime}$ onto an edge e $\in E\left(\Sigma^{\prime}\right)$ of the same type.

Proof: Using induction with respect to $|\Sigma|$, we may assume, without loss of generality, that $\Sigma=\Sigma^{\prime} \cup\{S\}$ holds for some single split $S=\{A, B\}$ in $\Sigma-\Sigma^{\prime}$. Then, at least one of the two extensions $\psi_{A}, \psi_{B}$ of $\psi$ in $V^{*}(\Sigma)$ defined by putting $\psi_{A}(S):=A$ and $\psi_{B}(S):=B$ must be contained in $V(\Sigma)$ : Indeed, if $\psi_{A} \notin V^{*}(\Sigma)$ would hold, there would exist some $S^{\prime} \in \Sigma^{\prime}$ with $\psi_{A}(S) \cap \psi_{A}\left(S^{\prime}\right)=A \cap \psi\left(S^{\prime}\right)=\emptyset$. In this case, however, $\psi_{B}(S) \cap \psi_{B}\left(S^{\prime \prime}\right)=B \cap \psi\left(S^{\prime \prime}\right) \neq \emptyset$ would hold for all $S^{\prime \prime} \in \Sigma^{\prime}$ since $B \cap \psi\left(S^{\prime \prime}\right)=\emptyset$ for some $S^{\prime \prime} \in \Sigma^{\prime}$ would imply $\psi\left(S^{\prime}\right) \cap \psi\left(S^{\prime \prime}\right) \subseteq B \cap A=\emptyset$ in contradiction to $\psi \in V\left(\Sigma^{\prime}\right)$. So, $\psi_{B} \in V^{*}(\Sigma)$ must hold in this case. I

Corollary 2.6. Given any two distinct splits $S, S^{\prime}$ in a split system $\Sigma$, there exist always two maps $\psi, \psi^{\prime} \in V(\Sigma)$ with $S, S^{\prime} \in \Delta\left(\psi, \psi^{\prime}\right)$.

Proof: This follows directly from the last lemma as it obviously holds in case $\Sigma=\left\{S, S^{\prime}\right\}$.

\section{Some Graph-Theoretical Observations}

To establish our main results, we will make use of the following simple and purely graph-theoretical observations: 
Suppose that $U$ and $V$ are two sets and that $R \subseteq U \times V$ is a binary relation. Let $\Gamma(R)$ denote the bipartite graph with vertex set the "disjoint amalgamation" $U \amalg V$ of $U$ and $V$, and edge set $E(R):=\{\{u, v\}$ : $u \in U, v \in V,(u, v) \in R\}$, and define graphs $\Gamma(R \mid U):=\left(U,\left\{\left\{u_{1}, u_{2}\right\} \in\right.\right.$ $\left.\left.\left(\begin{array}{l}U \\ 2\end{array}\right): \exists_{v \in V}\left(u_{1}, v\right),\left(u_{2}, v\right) \in R\right\}\right)$ and $\Gamma(R \mid V):=\left(V,\left\{\left\{v_{1}, v_{2}\right\} \in\left(\begin{array}{c}V \\ 2\end{array}\right)\right.\right.$ : $\left.\left.\exists_{u \in U}\left(u, v_{1}\right),\left(u, v_{2}\right) \in R\right\}\right)$. Let $\pi_{0}(R), \pi_{0}(R \mid U)$ and $\pi_{0}(R \mid V)$ denote the connected components of $\Gamma(R), \Gamma(R \mid U)$ and $\Gamma(R \mid V)$, respectively. Then, the following holds:

Lemma 3.1. Given two sets $U$ and $V$ and a binary relation $R \subseteq U \times V$ such that the associated bipartite graph $\Gamma(R)$ has no isolated vertices, the embeddings $\iota_{U}: U \rightarrow U \amalg V: u \mapsto u$ and $\iota_{V}: V \rightarrow U \amalg V: v \mapsto v$ induce bijections between the sets $\pi_{0}(R \mid U)$ and $\pi_{0}(R \mid V)$ and the set $\pi_{0}(R)$ of connected components of the graph $\Gamma(R)$. I.e., with obvious notational conventions, we have a commutative diagram of bijections:

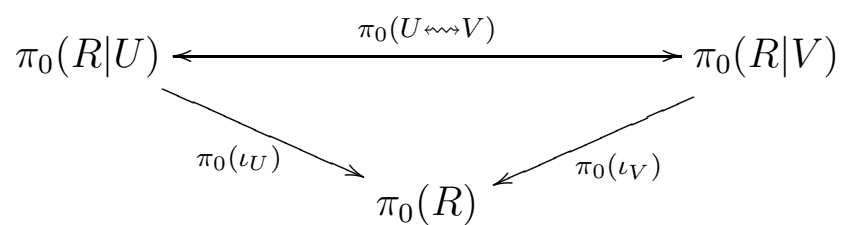

In particular, given two subsets $A \in \pi_{0}(R \mid U)$ and $B \in \pi_{0}(R \mid V)$ of $U$ and $V$, respectively, the following assertions are equivalent:

(i) $\pi_{0}(U \rightsquigarrow V)(A)=B$,

(ii) $(A \times B) \cap R \neq \emptyset$,

(iii) $B=\left\{v \in V: \exists_{a \in A}(a, v) \in R\right\}$,

(iv) $A=\left\{u \in U: \exists_{b \in B}(u, b) \in R\right\}$.

Proof: All this is quite obvious: The maps $\pi_{0}\left(\iota_{U}\right)$ and $\pi_{0}\left(\iota_{V}\right)$ must be surjective as $\Gamma(R)$ is supposed to have no isolated vertices. And they must be injective because any path in $\Gamma(R)$ connecting two vertices $u_{1}, u_{2} \in U$ (or $v_{1}, v_{2} \in V$ ) gives rise to a path connecting these two vertices in $\Gamma(R \mid U)$ (or in $\Gamma(R \mid V)$, respectively).

Now, assume that $U^{\prime}$ and $V^{\prime}$ are two further sets, and that $\alpha: U^{\prime} \rightarrow U$ and $\beta: V^{\prime} \rightarrow V$ are two maps such that there exists

(M1) for all $u_{1}, u_{2} \in U$ and $v \in V$ with $\left(u_{1}, v\right),\left(u_{2}, v\right) \in R$, some $v^{\prime} \in V^{\prime}$ with $\left(u_{1}, \beta\left(v^{\prime}\right)\right),\left(u_{2}, \beta\left(v^{\prime}\right)\right) \in R$ and, symmetrically,

(M2) for all $u \in U$ and $v_{1}, v_{2} \in V$ with $\left(u, v_{1}\right),\left(u, v_{2}\right) \in R$, some $u^{\prime} \in U^{\prime}$ with $\left(\alpha\left(u^{\prime}\right), v_{1}\right),\left(\alpha\left(u^{\prime}\right), v_{2}\right) \in R$.

Then, defining the binary relations

$$
\begin{gathered}
R^{\prime}:=R_{\alpha, \beta}:=\left\{\left(u^{\prime}, v^{\prime}\right) \in U^{\prime} \times V^{\prime}:\left(\alpha\left(u^{\prime}\right), \beta\left(v^{\prime}\right)\right) \in R\right\}, \\
R_{\alpha}:=\left\{\left(u^{\prime}, v\right) \in U^{\prime} \times V:\left(\alpha\left(u^{\prime}\right), v\right) \in R\right\},
\end{gathered}
$$

\footnotetext{
${ }^{1}$ Here, we make use of the fact that, according to category theory, the disjoint amalgamation of any two sets $U$ and $V$ is well defined up to canonical bijection whether or not $U$ and $V$ are disjoint, and can be constructed e.g. by considering the subset of the set $(U \cup V) \times\{1,2\}$ consisting of all $(w, i) \in(U \times\{1\}) \cup(V \times\{2\})$.
} 
and

$$
R_{\beta}:=\left\{\left(u, v^{\prime}\right) \in U \times V^{\prime}:(u, \beta(v)) \in R\right\},
$$

it is easily seen that the following holds:

- The graph $\Gamma\left(R_{\alpha} \mid V\right)$ coincides with the graph $\Gamma(R \mid V)$.

- The graph $\Gamma\left(R_{\beta} \mid U\right)$ coincides with the graph $\Gamma(R \mid U)$.

- The graph $\Gamma\left(R_{\alpha} \mid U^{\prime}\right)$ coincides with $\Gamma\left(R^{\prime} \mid U^{\prime}\right)$ as well as with the graph induced by $\Gamma(R \mid U)$ and $\alpha$ on $U^{\prime}$.

- The graph $\Gamma\left(R_{\beta} \mid V^{\prime}\right)$ coincides with $\Gamma\left(R^{\prime} \mid V^{\prime}\right)$ as well as with the graph induced by $\Gamma(R \mid V)$ and $\beta$ on $V^{\prime}$.

Furthermore, all the corresponding maps must induce bijections on the level of connected components, i.e., we have

Corollary 3.2. Continuing with the assumptions introduced above as well as in Lemma 3.1 and using obvious notational conventions, we have the following commutative diagram in which all maps are bijections:

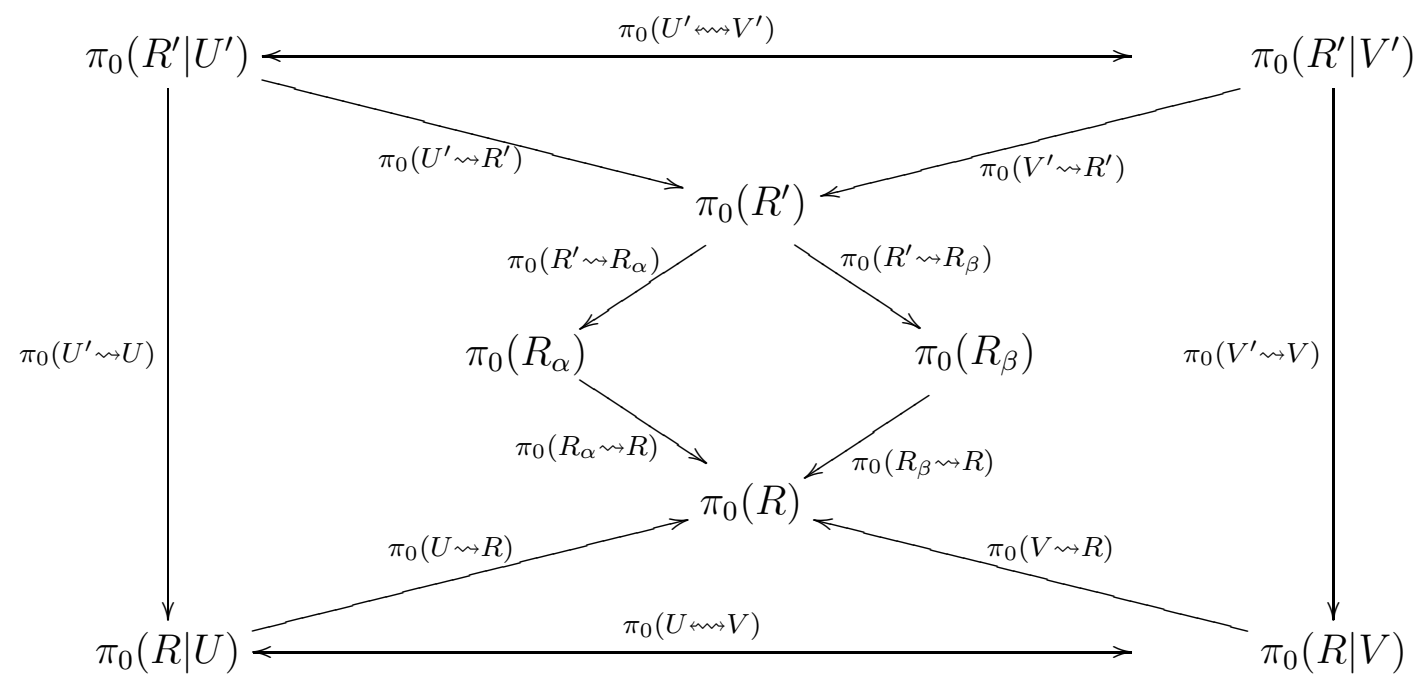

In particular, given four subsets $A^{\prime} \in \pi_{0}\left(R^{\prime} \mid U^{\prime}\right), A \in \pi_{0}(R \mid U)$, $B^{\prime} \in \pi_{0}\left(R^{\prime} \mid V^{\prime}\right)$, and $B \in \pi_{0}(R \mid V)$ of $U^{\prime}, U, V^{\prime}$ and $V$, respectively, the following holds:

(i) $\pi_{0}\left(U^{\prime} \longleftrightarrow V^{\prime}\right)\left(A^{\prime}\right)=B^{\prime} \Longleftrightarrow\left(A^{\prime} \times B^{\prime}\right) \cap R^{\prime} \neq \emptyset \Longleftrightarrow B^{\prime}=$ $\left\{v^{\prime} \in V^{\prime}: \exists_{a^{\prime} \in A^{\prime}}\left(a^{\prime}, v^{\prime}\right) \in R^{\prime}\right\}$

(ii) $\pi_{0}(\alpha)\left(A^{\prime}\right)=A \Longleftrightarrow \alpha\left(A^{\prime}\right) \cap A \neq \emptyset \Longleftrightarrow \alpha\left(A^{\prime}\right) \subseteq A \Longleftrightarrow$ $A=\left\{u \in U: \exists_{a^{\prime} \in A^{\prime}, v \in V}(u, v) \in R\right.$ and $\left.\left(a^{\prime}, v\right) \in R_{\alpha}\right\}$

(iii) $\left(\pi_{0}(\beta) \circ \pi_{0}\left(U^{\prime} \longleftrightarrow V^{\prime}\right)\right)\left(A^{\prime}\right)=B \Longleftrightarrow\left(\alpha\left(A^{\prime}\right) \times B\right) \cap R \neq \emptyset \Longleftrightarrow$ $B=\left\{v \in V: \exists_{a^{\prime} \in A^{\prime}}\left(\alpha\left(a^{\prime}\right), v\right) \in R\right\}$

Proof: It follows from Lemma 3.1 that all non-vertical maps in the above diagram must be bijections. Moreover, if either $\alpha$ or $\beta$ is the identity map, at least one of the vertical maps must also be a bijection in which case all maps must be bijections. So, the general case follows by concatenating the diagram obtained for the pair $\left(\operatorname{id}_{U}, \beta\right)$ with the diagram obtained for the pair $\left(\alpha, \mathrm{id}_{V^{\prime}}\right)$ and applying what we know 
about the individual binary relations $R^{\prime}, R_{\alpha}$, and $R$.

\section{Some Characterizations of Cut Vertices}

In this section, we will provide some characterizations of the cut vertices in the Buneman graph. Some of these closely resemble the characterization of cut points in the tight span of a metric space in terms of certain finite graphs given in [13]. More precisely, we will establish the following result:

Theorem 4.1. Assume as above that $X$ is a finite set, that $\Sigma$ is a system of $X$-splits, and that $\phi$ is a map in $V(\Sigma)$. Then, the following assertions are equivalent:

(i) $\phi$ is a cut vertex of $\mathcal{B}(\Sigma)$.

(ii) There exists a bipartition of $\Sigma^{(\phi)}:=\{S \in \Sigma: \phi(S) \in \min (\phi[\Sigma])\}$ into two disjoint non-empty subsets $\Sigma_{1}^{(\phi)}$ and $\Sigma_{2}^{(\phi)}$ such that any two splits $S_{1} \in \Sigma_{1}^{(\phi)}$ and $S_{2} \in \Sigma_{2}^{(\phi)}$ are compatible.

(iii) $\Sigma^{(\phi)}$ has a non-empty intersection with at least two distinct connected components of the incompatibility graph $\Gamma(\Sigma)$ of $\Sigma$, as defined in the introduction.

(iv) There exists a bipartition of $\Sigma$ into two disjoint non-empty subsets $\Sigma_{1}$ and $\Sigma_{2}$ such that $\phi\left(S_{1}\right) \cup \phi\left(S_{2}\right)=X$ holds for any two splits $S_{1} \in \Sigma_{1}$ and $S_{2} \in \Sigma_{2}$.

(v) There exists a bipartition of $X^{(\phi)}:=\left\{x \in X: \phi \neq \phi_{x}\right\}$ into two disjoint non-empty subsets $X_{1}$ and $X_{2}$ such that $X_{1} \subseteq \phi(S)$ or $X_{2} \subseteq \phi(S)$ holds for all $S \in \Sigma$ or, equivalently, for all $S \in \Sigma^{(\phi)}$.

(vi) There exists a bipartition of $V^{(\phi)}=V^{(\phi)}(\Sigma):=V(\Sigma)-\{\phi\}$ into two disjoint non-empty subsets $V_{1}$ and $V_{2}$ such that $\Delta\left(\phi, \psi_{1}\right) \cap$ $\Delta\left(\phi, \psi_{2}\right)=\emptyset$ holds for all $\psi_{1} \in V_{1}$ and $\psi_{2} \in V_{2}$.

To establish Theorem 4.1, we apply Corollary 3.2 as follows: With $X, \Sigma$, and $\phi$ as in the theorem, we put $U:=\Sigma, V:=V^{(\phi)}, U^{\prime}:=\Sigma^{(\phi)}$, $V^{\prime}:=X^{(\phi)}$. Further, we denote by $\alpha$ the embedding of $\Sigma^{(\phi)}$ into $\Sigma$ and by $\beta$ the map $X^{(\phi)} \rightarrow V^{(\phi)}: x \mapsto \phi_{x}$. And we put $R:=R^{(\phi)}:=$ $\left\{(S, \psi) \in \Sigma \times V^{(\phi)}: S \in \Delta(\phi, \psi)\right\}$.

It is easily checked that all the requirements needed for applying Corollary 3.2 are satisfied. For example, the maps $\alpha$ and $\beta$ satisfy Properties (M1) and (M2): Indeed, given two maps $\psi_{1}, \psi_{2} \in V^{(\phi)}$, and some split $S \in \Sigma$ with $S \in \Delta\left(\phi, \psi_{1}\right) \cap \Delta\left(\phi, \psi_{2}\right)$, there exists a split in $S^{\prime} \in \Sigma^{(\phi)}$ with $S^{\prime} \in \Delta\left(\phi, \psi_{1}\right) \cap \Delta\left(\phi, \psi_{2}\right)$ as $\psi_{1}(S)=\psi_{2}(S) \neq \phi(S) \supseteq$ $\phi\left(S^{\prime}\right)$ for some $S, S^{\prime} \in \Sigma$ implies $\phi\left(S^{\prime}\right) \cap \psi_{1}(S)=\phi\left(S^{\prime}\right) \cap \psi_{2}(S)=\emptyset$ and, hence, $\psi_{1}\left(S^{\prime}\right)=\psi_{2}\left(S^{\prime}\right) \neq \phi\left(S^{\prime}\right)$. And if $S, S^{\prime} \in \Delta(\phi, \psi)$ holds for some $S, S^{\prime} \in \Sigma$ and $\psi \in V^{(\phi)}$, then $\psi(S) \cap \psi\left(S^{\prime}\right) \neq \emptyset$ implies that there is some $x \in X^{(\phi)}$ with $S, S^{\prime} \in \Delta\left(\phi, \phi_{x}\right)$ as this must hold for any $x \in \psi(S) \cap \psi\left(S^{\prime}\right)$. 
Now, with $W$ denoting any of the sets $\Sigma^{(\phi)}, X^{(\phi)}, \Sigma$, and $V^{(\phi)}$ or their cartesian products $\Sigma^{(\phi)} \times X^{(\phi)}, \Sigma^{(\phi)} \times V^{(\phi)}, \Sigma \times X^{(\phi)}$, or $\Sigma \times V^{(\phi)}$, let $\Gamma_{\phi}(W)$ denote the corresponding graph with vertex set $W$ whose edge set $E_{\phi}(W)$ is defined in terms of the binary relations $R^{(\phi)}, R_{\alpha}^{(\phi)}, R_{\beta}^{(\phi)}$, and $R_{\alpha, \beta}^{(\phi)}$ - so, for example, the edge set $E_{\phi}(\Sigma)$ of $\Gamma_{\phi}(\Sigma)$ is the set

$$
E_{\phi}(\Sigma)=\left\{\left\{S_{1}, S_{2}\right\} \in\left(\begin{array}{c}
\Sigma \\
2
\end{array}\right): \exists_{\psi \in V^{(\phi)}} S_{1}, S_{2} \in \Delta(\phi, \psi)\right\} .
$$

Note that in what comes below, we shall derive explicit descriptions of the edge sets of the graphs $\Gamma_{\phi}(\Sigma), \Gamma_{\phi}\left(\Sigma^{(\phi)}\right), \Gamma_{\phi}\left(X^{(\phi)}\right)$, and $\Gamma_{\phi}\left(V^{(\phi)}\right)$ in (2), (3), (4), and (5), respectively. It may be helpful for the reader to take a look at these explicit descriptions before proceeding.

In addition, to further simplify notation, put $\pi_{\phi}(W):=\pi_{0}\left(\Gamma_{\phi}(W)\right)$ and, for any pair of distinct vertex sets $W, W^{\prime}$ as above, denote by $\pi_{\phi}\left(W^{\prime} \rightsquigarrow W\right)$ the induced bijection from $\pi_{\phi}\left(W^{\prime}\right)$ onto $\pi_{\phi}(W)$.

Then, Corollary 3.2 yields the following diagram of canonical bijections:

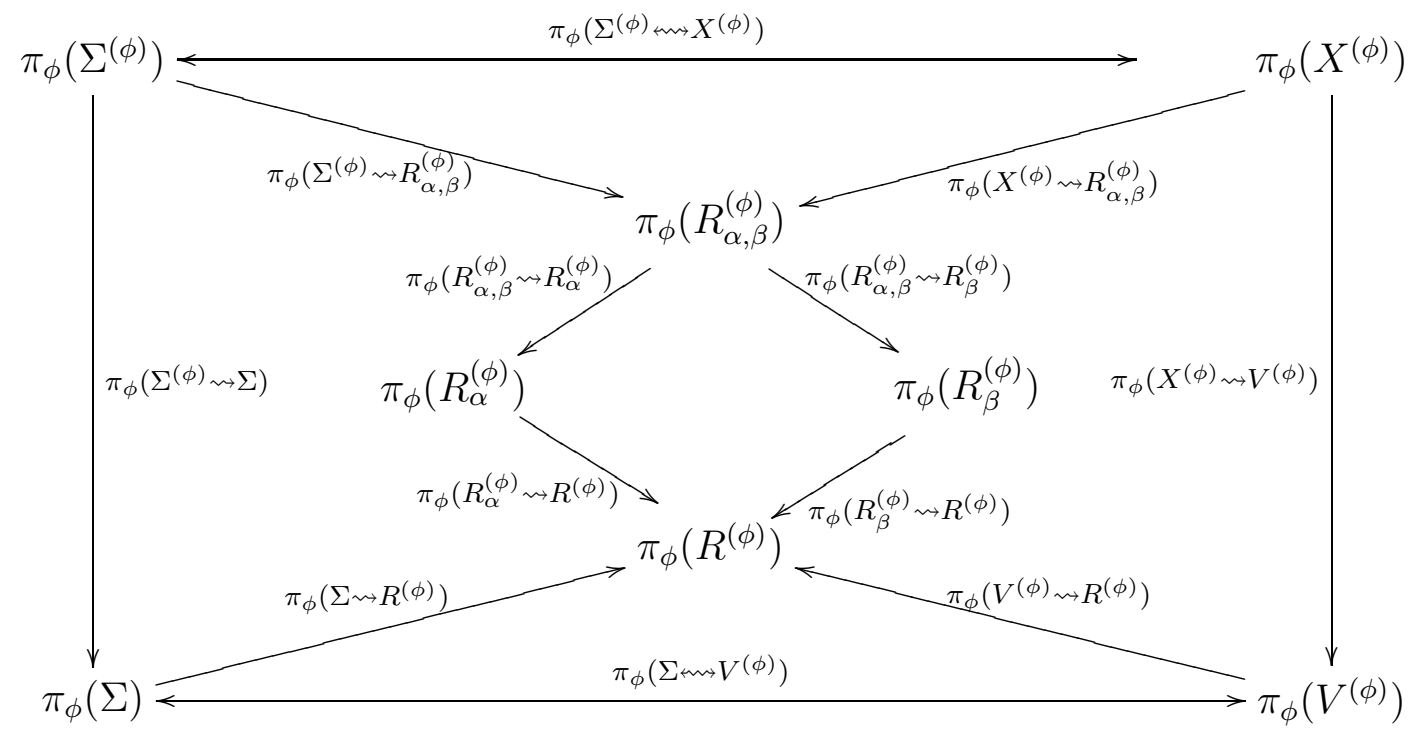

Now, note that the graph $\Gamma_{\phi}\left(V^{(\phi)}\right)$ contains the induced subgraph $\mathcal{B}^{(\phi)}(\Sigma)$ of $\mathcal{B}(\Sigma)=(V(\Sigma), E(\Sigma))$ with vertex set $V^{(\phi)}$ — the graph that, by definition, is disconnected if and only if $\phi$ is a cut vertex (of $\mathcal{B}(\Sigma)$ ): Indeed, any edge $\left\{\psi, \psi^{\prime}\right\} \in E(\Sigma)$ with $\psi, \psi^{\prime} \neq \phi$ must also be an edge in $\Gamma_{\phi}\left(V^{(\phi)}\right)$ as $\left\{\psi, \psi^{\prime}\right\} \in E(\Sigma)$ implies that either $\Delta(\psi, \phi) \subset \Delta\left(\psi^{\prime}, \phi\right)$ or $\Delta\left(\psi^{\prime}, \phi\right) \subset \Delta(\psi, \phi)$ must hold. So, $\psi, \psi^{\prime} \neq \phi$ implies that some split $S \in \Delta(\psi, \phi) \cap \Delta\left(\psi^{\prime}, \phi\right)$ must exist.

Furthermore, the embedding of $\mathcal{B}^{(\phi)}(\Sigma)$ into $\Gamma_{\phi}\left(V^{(\phi)}\right)$ induces a bijection between the corresponding sets of connected components $\pi_{0}\left(\mathcal{B}^{(\phi)}(\Sigma)\right)$ and $\pi_{\phi}\left(V^{(\phi)}\right)$ (the latter being the set in the bottom right corner of the above commutative diagram): Indeed, given any two maps $\psi, \psi^{\prime} \in V^{(\phi)}$ that form an edge in $\Gamma_{\phi}\left(V^{(\phi)}\right)$, there must exist some split $S \in \Delta(\psi, \phi) \cap$ 
$\Delta\left(\psi^{\prime}, \phi\right)$ implying that $S \in \Delta\left(\psi_{i}, \phi\right)$ and, hence, that also $\psi_{i} \neq \phi$ must hold for every map $\psi_{i}$ in any shortest path $\psi_{0}:=\psi, \psi_{1}, \ldots, \psi_{k}:=\psi^{\prime}$ from $\psi$ to $\psi^{\prime}$ in $\mathcal{B}(\Sigma)$. Therefore, $\psi$ and $\psi^{\prime}$ must also be contained in the same connected component of $\mathcal{B}^{(\phi)}(\Sigma)$. In consequence, any connected component of $\Gamma_{\phi}\left(V^{(\phi)}\right)$ must be contained in and, hence, coincide with connected component of $\mathcal{B}^{(\phi)}(\Sigma)$.

Thus, in view of the above diagram, a map $\phi \in V(\Sigma)$ is a cut vertex of $\mathcal{B}(\Sigma)$ if and only if either one of the eight graphs $\Gamma_{\phi}(W)$ with $W=$ $\Sigma^{(\phi)}, X^{(\phi)}, \Sigma, V^{(\phi)}, \Sigma^{(\phi)} \times X^{(\phi)}, \Sigma^{(\phi)} \times V^{(\phi)}, \Sigma \times X^{(\phi)}$, or $\Sigma \times V^{(\phi)}$ as above is disconnected.

So, denoting the connected component of $\Gamma_{\phi}(W)$ containing a given vertex $w \in W$ by $\Gamma_{\phi}(W, w)$, Theorem 4.1 follows immediately from the following observations:

(i) $\Longleftrightarrow$ (iv): The definition of the edge set $E_{\phi}(\Sigma)$ implies that two distinct splits $S, S^{\prime} \in \Sigma$ form an edge in $E_{\phi}(\Sigma)$ if and only if there is some $x \in \overline{\phi(S)} \cap \overline{\phi\left(S^{\prime}\right)}$. I.e., we have

$$
E_{\phi}(\Sigma)=\left\{\left\{S, S^{\prime}\right\} \in\left(\begin{array}{l}
\Sigma \\
2
\end{array}\right): \phi(S) \cup \phi\left(S^{\prime}\right) \neq X\right\} .
$$

So, $\Gamma_{\phi}(\Sigma)$ is disconnected if and only if there exists a bipartition of $\Sigma$ into two disjoint non-empty subsets $\Sigma_{1}$ and $\Sigma_{2}$ such that $\phi\left(S_{1}\right) \cup \phi\left(S_{2}\right)=$ $X$ holds for any two splits $S_{1} \in \Sigma_{1}$ and $S_{2} \in \Sigma_{2}$. This establishes the equivalence of (i) and (iv) in Theorem 4.1.

It follows also that the incompatibility graph $\Gamma(\Sigma)$ of $\Sigma$ is a subgraph of $\Gamma_{\phi}(\Sigma)$. So, $\Gamma_{\phi}(\Sigma)$ must be connected for every map $\phi \in V(\Sigma)$ whenever the incompatibility graph $\Gamma(\Sigma)$ is connected. In consequence, $\mathcal{B}^{(\phi)}(\Sigma)$ must be 2-connected in this case, and, more generally, every connected component in $\pi_{0}(\Sigma)=\pi_{0}(\Gamma(\Sigma))$ of $\Gamma(\Sigma)$ must be contained in a connected component of $\Gamma_{\phi}(\Sigma)$. Equivalently, every connected component of $\Gamma_{\phi}(\Sigma)$ is a disjoint union of connected components of $\Gamma(\Sigma)$. The corresponding canonical surjection from $\pi_{0}(\Sigma)$ onto $\pi_{\phi}(\Sigma)=\pi_{0}\left(\Gamma_{\phi}(\Sigma)\right)$ that maps any connected component $\Gamma(\Sigma, S)$ of $\Gamma(\Sigma)$ containing a split $S \in \Sigma$ onto the corresponding connected component $\Gamma_{\phi}(\Sigma, S)$ of $\Gamma_{\phi}(\Sigma)$ containing $S$ will henceforth be denoted by $\pi_{\phi}(\rightarrow)$.

(i) $\Longleftrightarrow$ (ii): This follows immediately from the following observation:

Lemma 4.2. With $X, \Sigma$, and $\phi$ as above, two splits $S, S^{\prime} \in \Sigma^{(\phi)}$ form an edge in the induced subgraph $\Gamma_{\phi}\left(\Sigma^{(\phi)}\right)$ of $\Gamma_{\phi}(\Sigma)$ with vertex set $\Sigma^{(\phi)}$ if and only if they are incompatible. I.e., we have

$$
E_{\phi}\left(\Sigma^{(\phi)}\right)=\left\{\left\{S, S^{\prime}\right\} \in\left(\begin{array}{c}
\Sigma^{(\phi)} \\
2
\end{array}\right): S \text { and } S^{\prime} \text { are incompatible }\right\} .
$$

Proof of Lemma: Indeed, if two distinct $S, S^{\prime} \in \Sigma^{(\phi)}$ are incompatible, they form an edge in $\Gamma_{\phi}(\Sigma)$. Conversely, if they form an edge in $\Gamma_{\phi}(\Sigma)$, i.e., if $\overline{\phi(S)} \cap \overline{\phi\left(S^{\prime}\right)} \neq \emptyset$ holds, they must be incompatible. This follows 
as $\phi(S) \cap \phi\left(S^{\prime}\right) \neq \emptyset$ holds for any two splits $S, S^{\prime} \in \Sigma$ in view of $\phi \in V(\Sigma)$, and $\overline{\phi(S)} \cap \phi\left(S^{\prime}\right) \neq \emptyset$ holds for any two distinct splits $S \in \Sigma^{(\phi)}$ and $S^{\prime} \in \Sigma$.

(i) $\Longleftrightarrow$ (iii): Lemma 4.2 implies that $\Gamma_{\phi}\left(\Sigma^{(\phi)}\right)$ can be viewed as the induced subgraph of the incompatibility graph $\Gamma(\Sigma)$ with vertex set $\Sigma^{(\phi)} \subseteq \Sigma$. This implies that the embedding $\Sigma^{(\phi)} \rightarrow \Sigma$ induces a welldefined map $\pi_{\phi}\left(\Sigma^{(\phi)}\right) \rightarrow \pi_{0}(\Sigma): \Gamma_{\phi}\left(\Sigma^{(\phi)}, S\right) \mapsto \Gamma(\Sigma, S)$ that we denote, for short, by $\pi_{\phi}(\longmapsto)$. Moreover, the composition

$$
\pi_{\phi}(\rightarrow) \circ \pi_{\phi}(\longmapsto): \pi_{\phi}\left(\Sigma^{(\phi)}\right) \rightarrow \pi_{0}(\Sigma) \rightarrow \pi_{\phi}(\Sigma)
$$

maps, for any split $S \in \Sigma^{(\phi)}$, the connected component $\Gamma_{\phi}\left(\Sigma^{(\phi)}, S\right)$ onto the connected component $\Gamma_{\phi}(\Sigma, S)$ and, hence, coincides with the bijection $\pi_{\phi}\left(\Sigma^{(\phi)} \rightsquigarrow \Sigma\right): \Gamma_{\phi}\left(\Sigma^{(\phi)}, S\right) \mapsto \Gamma_{\phi}(\Sigma, S)$ induced by the embedding $\alpha$ of $\Sigma^{(\phi)}$ into $\Sigma$. In turn, this implies

Lemma 4.3. The map $\pi_{\phi}(\longmapsto)$ is always injective, that is, any two splits $S, S^{\prime} \in \Sigma^{(\phi)}$ can be connected by a sequence of pairwise incompatible splits in $\Sigma^{(\phi)}$ whenever they can be connected by such a sequence of pairwise incompatible splits in $\Sigma$.

This clearly establishes that (i) $\Longleftrightarrow$ (iii) holds, as claimed.

(i) $\Longleftrightarrow(\mathrm{v}) \Longleftrightarrow(\mathrm{vi})$ : We now establish the equivalence of the first and the last two assertions in Theorem 4.1. It suffices to note that two elements $x_{1}, x_{2}$ in $X^{(\phi)}$ are not connected by an edge in $\Gamma_{\phi}\left(X^{(\phi)}\right)$ if and only if $x_{1} \in \phi(S)$ or $x_{2} \in \phi(S)$ holds for all $S \in \Sigma$ or, equivalently, for all $S \in \Sigma^{(\phi)}$. I.e., we have

$$
\begin{aligned}
& E_{\phi}\left(X^{(\phi)}\right) \\
& =\left\{\left\{x_{1}, x_{2}\right\} \in\left(\begin{array}{c}
X^{(\phi)} \\
2
\end{array}\right): \exists_{\left.S \in \Sigma \text { (or } \Sigma^{(\phi)}\right)} x_{1}, x_{2} \notin \phi(S)\right\} \\
& =\left\{\left\{x_{1}, x_{2}\right\} \in\left(\begin{array}{c}
X^{(\phi)} \\
2
\end{array}\right): D\left(\phi_{x_{1}}, \phi_{x_{2}}\right)<D\left(\phi_{x_{1}}, \phi\right)+D\left(\phi, \phi_{x_{2}}\right)\right\} .
\end{aligned}
$$

Moreover, two maps $\psi_{1}, \psi_{2}$ in $V^{(\phi)}$ are not connected by an edge in $\Gamma_{\phi}\left(V^{(\phi)}\right)$ if and only if $\phi(S)=\psi_{1}(S)$ or $\phi(S)=\psi_{2}(S)$ holds for all $S \in \Sigma$ or, equivalently, for all $S \in \Sigma^{(\phi)}$. I.e., we have

$$
\begin{aligned}
& E_{\phi}\left(V^{(\phi)}\right) \\
& =\left\{\left\{\psi_{1}, \psi_{2}\right\} \in\left(\begin{array}{c}
V^{(\phi)} \\
2
\end{array}\right): D\left(\psi_{1}, \psi_{2}\right)<D\left(\psi_{1}, \phi\right)+D\left(\phi, \psi_{2}\right)\right\} \\
& =\left\{\left\{\psi_{1}, \psi_{2}\right\} \in\left(\begin{array}{c}
V^{(\phi)} \\
2
\end{array}\right): \exists_{S \in \Sigma\left(\text { or } \Sigma^{(\phi)}\right)} \psi_{1}(S)=\psi_{2}(S) \neq \phi(S)\right\} .
\end{aligned}
$$

This finishes the proof of Theorem 4.1. 
It is worth noting in this context that our approach implies also that, given a map $\phi \in V(\Sigma)$, the following assertions are equivalent:

(i) $\phi$ is a cut vertex.

(ii) There exists a bipartition $\left\{\Sigma_{1}, \Sigma_{2}\right\}$ of $\Sigma$ and a bipartition $\left\{V_{1}, V_{2}\right\}$ of $V^{(\phi)}$ such that $\phi(S)=\psi(S)$ holds for all $S \in \Sigma_{1}$ and $\psi \in V_{2}$, and for all $S \in \Sigma_{2}$ and $\psi \in V_{1}$.

(iii) There exists a bipartition $\left\{\Sigma_{1}, \Sigma_{2}\right\}$ of $\Sigma^{(\phi)}$ and a bipartition $\left\{X_{1}, X_{2}\right\}$ of $X^{(\phi)}$ such that $x \in \phi(S)$ holds for all $S \in \Sigma_{1}$ and $x \in X_{2}$, and for all $S \in \Sigma_{2}$ and $x \in X_{1}$.

For example, for the map $\phi$ in Figure1, the bipartition of $\Sigma_{8}$ is $\left\{\left\{S_{67}, S_{78}\right\}, \Sigma-\right.$ $\left.\left\{S_{67}, S_{78}\right\}\right\}$. Moreover, we have $\Sigma^{(\phi)}=\left\{S_{1235}, S_{1234}, S_{45}, S_{78}, S_{67}\right\}$, and the corresponding bipartition is given by $\left\{\left\{S_{67}, S_{78}\right\}, \Sigma^{(\phi)}-\left\{S_{67}, S_{78}\right\}\right\}$.

It is also worth noting that the various images of the connected components in the sets $\pi_{\phi}(\ldots)$ relative to the respective bijections considered above can be described as follows. For $\phi, \psi \in V^{*}(\Sigma)$, we put

$$
\Delta_{\min }(\psi \mid \phi):=\{S \in \Delta(\phi, \psi): \psi(S) \in \min (\psi[\Delta(\phi, \psi)])\}
$$

where $\min (\psi[\Delta(\phi, \psi)])$ denotes the set of (inclusion-)minimal subsets in the image $\psi[\Delta(\phi, \psi)]$ of $\Delta(\phi, \psi)$ relative to $\psi$.

Proposition 4.4. Given any four connected components $\Sigma_{0}^{\prime} \in \pi_{\phi}\left(\Sigma^{(\phi)}\right)$, $\Sigma_{0} \in \pi_{\phi}(\Sigma), X_{0} \in \pi_{\phi}\left(X^{(\phi)}\right)$, and $V_{0} \in \pi_{\phi}\left(V^{(\phi)}\right)$, the following holds:

(i) $\pi_{\phi}\left(\Sigma^{(\phi)} \rightsquigarrow \Sigma\right)\left(\Sigma_{0}^{\prime}\right)=\Sigma_{0} \Longleftrightarrow \Sigma_{0}^{\prime} \subseteq \Sigma_{0} \Longleftrightarrow \Sigma_{0}^{\prime}=\Sigma_{0} \cap$ $\Sigma^{(\phi)} \Longleftrightarrow \Sigma_{0}=\left\{S \in \Sigma: \phi(S) \cup \phi\left(S^{\prime}\right) \neq X\right.$ for some $\left.S^{\prime} \in \Sigma_{0}^{\prime}\right\}$.

(ii) $\pi_{\phi}\left(\Sigma^{(\phi)} \rightsquigarrow X^{(\phi)}\right)\left(\Sigma_{0}^{\prime}\right)=X_{0} \Longleftrightarrow X_{0}=\left\{x \in X^{(\phi)}: \Delta_{\min }\left(\phi \mid \phi_{x}\right) \subseteq\right.$ $\left.\Sigma_{0}^{\prime}\right\} \Longleftrightarrow X_{0}=\left\{x \in X^{(\phi)}: \Delta_{\min }\left(\phi \mid \phi_{x}\right) \cap \Sigma_{0}^{\prime} \neq \emptyset\right\} \Longleftrightarrow \Sigma_{0}^{\prime}=$ $\bigcup_{x \in X_{0}} \Delta_{\min }\left(\phi \mid \phi_{x}\right)$.

(iii) $\pi_{\phi}\left(\Sigma^{(\phi)} \rightsquigarrow V^{(\phi)}\right)\left(\Sigma_{0}^{\prime}\right)=V_{0} \Longleftrightarrow V_{0}=\left\{\psi \in V^{(\phi)}: \Delta_{\min }(\phi \mid \psi) \subseteq\right.$ $\left.\Sigma_{0}^{\prime}\right\} \Longleftrightarrow V_{0}=\left\{\psi \in V^{(\phi)}: \Delta_{\min }(\phi \mid \psi) \cap \Sigma_{0}^{\prime} \neq \emptyset\right\} \Longleftrightarrow V_{0} \supseteq$ $\left\{\phi^{S}: S \in \Sigma_{0}^{\prime}\right\} \Longleftrightarrow \Sigma_{0}^{\prime}=\bigcup_{\psi \in V_{0}} \Delta_{\min }(\phi \mid \psi) \Longleftrightarrow \Sigma_{0}^{\prime}=\{S \in$ $\left.\Sigma^{(\phi)}: \phi^{S} \in V_{0}\right\}$.

(iv) $\pi_{\phi}\left(\Sigma \rightsquigarrow X^{(\phi)}\right)\left(\Sigma_{0}\right)=X_{0} \Longleftrightarrow X_{0}=\left\{x \in X^{(\phi)}: \Delta\left(\phi, \phi_{x}\right) \subseteq\right.$ $\left.\Sigma_{0}\right\} \Longleftrightarrow X_{0}=\left\{x \in X^{(\phi)}: \Sigma_{0} \cap \Delta\left(\phi, \phi_{x}\right) \neq \emptyset\right\} \Longleftrightarrow \Sigma_{0}=$ $\bigcup_{x \in X_{0}} \Delta\left(\phi, \phi_{x}\right)$.

(v) $\pi_{\phi}\left(\Sigma \Longleftrightarrow V^{(\phi)}\right)\left(\Sigma_{0}\right)=V_{0} \Longleftrightarrow V_{0}=\left\{\psi \in V^{(\phi)}: \Delta(\phi, \psi) \subseteq\right.$ $\left.\Sigma_{0}\right\} \Longleftrightarrow V_{0}=\left\{\psi \in V^{(\phi)}: \Delta(\phi, \psi) \cap \Sigma_{0} \neq \emptyset\right\} \Longleftrightarrow \Sigma_{0}=$ $\bigcup_{\psi \in V_{0}} \Delta(\phi, \psi)$.

(vi) $\pi_{\phi}\left(X^{(\phi)} \rightsquigarrow V^{(\phi)}\right)\left(X_{0}\right)=V_{0} \Longleftrightarrow V_{0}=\left\{\psi \in V^{(\phi)}: D\left(\psi, \phi_{x}\right)<\right.$ $D(\psi, \phi)+D\left(\phi, \phi_{x}\right)$ for some $\left.x \in X_{0}\right\} \Longleftrightarrow V_{0}=\left\{\psi \in V^{(\phi)}\right.$ : $\Delta\left(\phi, \phi_{x}\right) \cap \Delta(\phi, \psi) \neq \emptyset$ for some $\left.x \in X_{0}\right\} \Longleftrightarrow X_{0}=\{x \in X$ : $\left.\phi_{x} \in V_{0}\right\}$. 
These assertions follow quite easily from our definitions. We leave their simple and straight-forward (yet sometimes a bit laborious) verification to the interested reader.

Note also that, continuing with the assumptions and notations of Theorem 4.1, our analysis implies the following corollary:

Corollary 4.5. The graph $\mathcal{B}(\Sigma)$ is 2-connected if and only if the incompatibility graph $\Gamma(\Sigma)$ is connected.

More generally, given any two maps $\psi, \psi^{\prime} \in V(\Sigma)$, one has $\Gamma_{\phi}\left(V^{(\phi)}, \psi\right)$ $=\Gamma_{\phi}\left(V^{(\phi)}, \psi^{\prime}\right)$ for all $\phi \in V(\Sigma)-\left\{\psi, \psi^{\prime}\right\}$ if and only if $\Delta\left(\psi, \psi^{\prime}\right) \subseteq \Sigma_{0}$ holds for some connected component $\Sigma_{0}$ of the incompatibility graph $\Gamma(\Sigma)$ of $\Sigma$. So, conversely, two maps $\psi, \psi^{\prime} \in V(\Sigma)$ are separated by some cut vertex $\phi \in V(\Sigma)$ if and only if $\Delta\left(\psi, \psi^{\prime}\right)$ has a non-empty intersection with at least two distinct connected components of $\Gamma(\Sigma)$.

Proof: We have already seen above that $\mathcal{B}(\Sigma)$ cannot contain a cut vertex in case $\Gamma(\Sigma)$ is connected.

Conversely, if $\Gamma(\Sigma)$ is not connected, one may choose any two splits $S, S^{\prime}$ in distinct connected components of $\Gamma(\Sigma)$ and then, according to Corollary 2.6, two maps $\psi, \psi^{\prime} \in V(\Sigma)$ with $S, S^{\prime} \in \Delta\left(\psi, \psi^{\prime}\right)$. Then, given any shortest path $\psi_{0}:=\psi, \psi_{1}, \ldots, \psi_{k}:=\psi^{\prime}$ from $\psi$ to $\psi^{\prime}$ in $\mathcal{B}(\Sigma)$, there must exist some $i$ in $\{1, \ldots, k-1\}$ such that the two splits in the one-split sets $\Delta\left(\psi_{i}, \psi_{i-1}\right)$ and $\Delta\left(\psi_{i}, \psi_{i+1}\right)$ are in distinct connected components of $\Gamma(\Sigma)$. Thus, $\psi_{i}$ must be a cut vertex in $\mathcal{B}(\Sigma)$.

Moreover, if $\Delta\left(\psi, \psi^{\prime}\right) \subseteq \Sigma_{0}$ holds for some connected component $\Sigma_{0}$ of the incompatibility graph $\Gamma(\Sigma)$ and $\phi$ is any map in $V(\Sigma)-\left\{\psi, \psi^{\prime}\right\}$, we must have $\Gamma_{\phi}\left(V^{(\phi)}, \psi\right)=\Gamma_{\phi}\left(V^{(\phi)}, \psi^{\prime}\right)$ : Indeed, Proposition $4.4(\mathrm{v})$ implies that $\Gamma_{\phi}\left(V^{(\phi)}, \psi\right) \neq \Gamma_{\phi}\left(V^{(\phi)}, \psi^{\prime}\right)$ holds for some maps $\psi, \psi^{\prime} \in$ $V^{(\phi)}$ if and only if the two sets $\Delta(\psi, \phi)$ and $\Delta\left(\phi, \psi^{\prime}\right)$ are contained in two distinct connected components of $\Gamma_{\phi}(\Sigma)$. In turn, this implies that $\Delta\left(\psi, \psi^{\prime}\right)$ must coincide with the disjoint union of $\Delta(\psi, \phi)$ and $\Delta\left(\phi, \psi^{\prime}\right)$. So, $\Delta\left(\psi, \psi^{\prime}\right)$ cannot be contained in a single connected component of $\Gamma_{\phi}(\Sigma)$ and, hence, even less in a single connected component of $\Gamma(\Sigma)$ in this case. Thus, given any two maps $\psi, \psi^{\prime} \in V(\Sigma), \Gamma_{\phi}\left(V^{(\phi)}, \psi\right)=$ $\Gamma_{\phi}\left(V^{(\phi)}, \psi^{\prime}\right)$ must hold indeed for any map $\phi$ in $V(\Sigma)-\left\{\psi, \psi^{\prime}\right\}$ in case $\Delta\left(\psi, \psi^{\prime}\right) \subseteq \Sigma_{0}$ holds for some connected component $\Sigma_{0}$ of the incompatibility graph $\Gamma(\Sigma)$.

Conversely, if $\Gamma_{\phi}\left(V^{(\phi)}, \psi\right)=\Gamma_{\phi}\left(V^{(\phi)}, \psi^{\prime}\right)$ holds for all $\phi \in V(\Sigma)-$ $\left\{\psi, \psi^{\prime}\right\}$, we must have $\Delta\left(\psi, \psi^{\prime}\right) \subseteq \Sigma_{0}$ for some connected component $\Sigma_{0}$ of the incompatibility graph $\Gamma(\Sigma)$. Indeed, choosing as above any path $\psi_{0}:=\psi, \psi_{1}, \ldots, \psi_{k}:=\psi^{\prime}$ from $\psi$ to $\psi^{\prime}$ in $\mathcal{B}(\Sigma)$, there would otherwise exist some $i$ in $\{1, \ldots, k-1\}$ such that the two splits in the one-split sets $\Delta\left(\psi_{i}, \psi_{i-1}\right)$ and $\Delta\left(\psi_{i}, \psi_{i+1}\right)$ are in distinct connected components of $\Gamma(\Sigma)$. In turn, this implies that, for $\phi:=\psi_{i}$, we would have $\Gamma_{\phi}\left(V^{(\phi)}, \psi_{i-1}\right) \neq \Gamma_{\phi}\left(V^{(\phi)}, \psi_{i+1}\right)$ in view of Proposition 4.4(iii), and the fact (cf. Lemma 4.3) that distinct connected components of $\Gamma(\Sigma)$ intersect $\Sigma^{(\phi)}$ in distinct connected components of $\Gamma_{\phi}\left(\Sigma^{(\phi)}\right)$ and, hence, 
$\Gamma_{\phi}\left(V^{(\phi)}, \psi\right)=\Gamma_{\phi}\left(V^{(\phi)}, \psi_{i-1}\right) \neq \Gamma_{\phi}\left(V^{(\phi)}, \psi_{i+1}\right)=\Gamma_{\phi}\left(V^{(\phi)}, \psi^{\prime}\right)$, a contradiction.

To conclude this section, we note that, essentially by definition, $\left(S, \phi^{S}\right) \in R^{(\phi)}$ holds for every $\phi \in V(\Sigma)$ and all $S \in \Sigma^{(\phi)}$. Thus, the obviously well-defined and injective map $\gamma: \Sigma^{(\phi)} \rightarrow V^{(\phi)}: S \mapsto \phi^{S}$ necessarily induces a map $\pi_{0}(\gamma)$ from $\pi_{\phi}\left(\Sigma^{(\phi)}\right)$ into $\pi_{\phi}\left(V^{(\phi)}\right)$ that must coincide with the bijection $\pi_{\phi}\left(\Sigma^{(\phi)} \rightsquigarrow V^{(\phi)}\right)$. So, we have a diagram of bijections all of which are "induced" by naturally defined maps between the corresponding vertex sets:

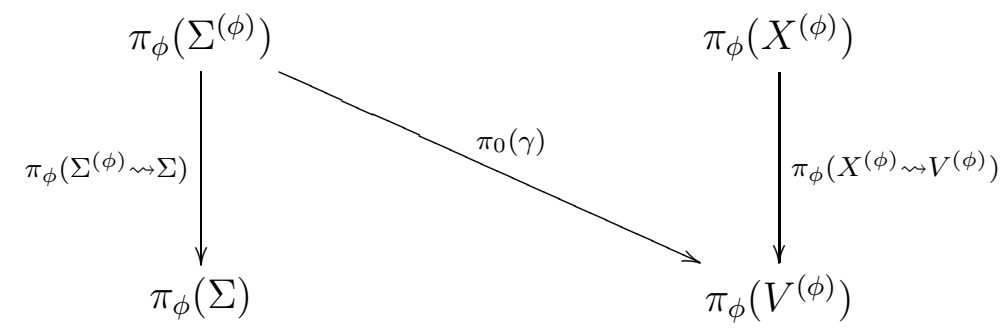

So, once established, these bijections could also have been used to define all the other bijections between the sets $\pi_{\phi}\left(\Sigma^{(\phi)}\right), \pi_{\phi}(\Sigma), \pi_{\phi}\left(X^{(\phi)}\right)$, and $\pi_{\phi}\left(V^{(\phi)}\right)$ which, however, we feel would have led to a much less transparent and natural approach.

\section{Blocks of the Buneman GRAPH}

For a collection $\Sigma$ of $X$-splits, we denote by $\operatorname{cut}(\Sigma)$ the set of all cut vertices of $\mathcal{B}(\Sigma)$ and, as mentioned in the introduction, by $B l(\Sigma)$ the set of all of its blocks. In this section, we will describe a canonical bijection between $B l(\Sigma)$ and $\pi_{0}(\Sigma)$, the set of connected components of the incompatibility graph of $\Sigma$.

To this end, note first that, given any two distinct compatible $X$ splits $S$ and $S^{\prime}$, there exists a unique subset $A \in S$ that we denote by $A\left(S \searrow S^{\prime}\right)$ such that $A \cap A^{\prime} \neq \emptyset$ and $A \cap B^{\prime} \neq \emptyset$ or, equivalently, $A^{\prime} \subset A$ or $B^{\prime} \subset A$ holds for the two subsets $A^{\prime}, B^{\prime}$ in $S^{\prime}$. Clearly, given $A \in S$ and $A^{\prime} \in S^{\prime}$, we have

(4.1) $A \cup A^{\prime}=X \Longleftrightarrow A=A\left(S \searrow S^{\prime}\right)$ and $A^{\prime}=A\left(S^{\prime} \searrow S\right)$.

(4.2) $A^{\prime} \subset A \Longleftrightarrow A=A\left(S \searrow S^{\prime}\right)$ and $A^{\prime} \neq A\left(S^{\prime} \searrow S\right)$.

(4.3) $A \subset A^{\prime} \Longleftrightarrow A \neq A\left(S \searrow S^{\prime}\right)$ and $A^{\prime}=A\left(S^{\prime} \searrow S\right)$.

(4.4) $A^{\prime} \cap A=\emptyset \Longleftrightarrow A \neq A\left(S \searrow S^{\prime}\right)$ and $A^{\prime} \neq A\left(S^{\prime} \searrow S\right)$.

(4.5) If $\phi \in V(\Sigma)$ and $S \in \Sigma^{(\phi)}$, then $\phi\left(S^{\prime}\right)=A\left(S^{\prime} \searrow S\right)$ must hold for every split $S^{\prime} \in \Sigma$ that is compatible with $S$.

Proof: Just apply (4.2) and (4.4) to $A:=\phi(S)$ and $A^{\prime}:=\phi\left(S^{\prime}\right)$, noting that neither $A^{\prime} \subset A$ nor $A^{\prime} \cap A=\emptyset$ can hold. 
Similarly, suppose that $S, S^{\prime}, S^{\prime \prime}$ are three distinct $X$-splits such that $S$ is compatible to $S^{\prime}$ and to $S^{\prime \prime}$. Then, as both $A\left(S^{\prime} \searrow S\right)$ and $A\left(S^{\prime \prime} \searrow\right.$ $S$ ) properly contain one of the two sets in $S$, we have

(4.6) $A\left(S^{\prime} \searrow S\right) \cap A\left(S^{\prime \prime} \searrow S\right) \neq \emptyset$, and

(4.7) $A\left(S \searrow S^{\prime}\right)=A\left(S \searrow S^{\prime \prime}\right)$ whenever $S^{\prime}$ and $S^{\prime \prime}$ are incompatible.

Proof: $A\left(S \searrow S^{\prime}\right) \neq A\left(S \searrow S^{\prime \prime}\right)$ would imply $A\left(S \searrow S^{\prime \prime}\right) \subset$ $A\left(S^{\prime} \searrow S\right)$ (apply (4.3) with $A:=A\left(S \searrow S^{\prime \prime}\right)$ ) and hence, by symmetry, also $A\left(S \searrow S^{\prime}\right) \subset A\left(S^{\prime \prime} \searrow S\right)$. Thus $X=A(S \searrow$ $\left.S^{\prime \prime}\right) \cup A\left(S \searrow S^{\prime}\right) \subseteq A\left(S^{\prime} \searrow S\right) \cup A\left(S^{\prime \prime} \searrow S\right)$, implying that $S^{\prime}$ and $S^{\prime \prime}$ must be compatible, a contradiction.

Thus, given any connected component $\Sigma_{0}$ of the incompatibility graph $\Gamma(\Sigma)$ of $\Sigma$ and any split $S \in \Sigma-\Sigma_{0}$, the two subsets $A\left(S \searrow S^{\prime}\right)$ and $A\left(S \searrow S^{\prime \prime}\right)$ in $S$ are well-defined and coincide for any two splits $S^{\prime}, S^{\prime \prime} \in \Sigma_{0}$. In consequence, we will also write $A\left(S \searrow \Sigma_{0}\right)$ for this subset in $S$.

Now, with $\Sigma$ a collection of $X$-splits as above, consider a connected component $\Sigma_{0} \in \pi_{0}(\Sigma)$ of the incompatibility graph $\Gamma(\Sigma)$ of $\Sigma$ and associate, to any map $\phi \in V^{*}\left(\Sigma_{0}\right)$, the map $\widetilde{\phi}: \Sigma \rightarrow \mathcal{P}(X)$ defined by putting

We claim

$$
\widetilde{\phi}(S):=\left\{\begin{array}{cc}
\phi(S) & \text { if } S \in \Sigma_{0}, \\
A\left(S \searrow \Sigma_{0}\right) & \text { else. }
\end{array}\right.
$$

Theorem 5.1. With $\Sigma$ and $\Sigma_{0}$ as above, we have $\widetilde{\phi} \in V(\Sigma)$ for every map $\phi \in V\left(\Sigma_{0}\right)$. Moreover, the corresponding embedding

$$
\Theta=\Theta_{\Sigma_{0}}: V\left(\Sigma_{0}\right) \rightarrow V(\Sigma): \phi \mapsto \widetilde{\phi}
$$

is actually an isometry from $V\left(\Sigma_{0}\right)$ into $V(\Sigma)$ that maps $V\left(\Sigma_{0}\right)$ onto a block

$$
B\left(\Sigma_{0}\right)=B_{\Sigma}\left(\Sigma_{0}\right):=\left\{\tilde{\phi}: \phi \in V\left(\Sigma_{0}\right)\right\}
$$

of $\mathcal{B}(\Sigma)$. Furthermore, we have

$$
\begin{aligned}
B\left(\Sigma_{0}\right) & =\left\{\phi \in V(\Sigma): \forall_{S \in \Sigma-\Sigma_{0}} \phi(S)=A\left(S \searrow \Sigma_{0}\right)\right\} \\
& =\left\{\phi \in V(\Sigma): \Sigma^{(\phi)} \cap \Sigma_{0} \neq \emptyset\right\}
\end{aligned}
$$

and, hence, also

$$
\left.B\left(\Sigma_{0}\right)=\left\{\phi \in V(\Sigma): \Delta\left(\phi, \phi_{0}\right) \subseteq \Sigma_{0}\right)\right\}
$$

for any map $\phi_{0} \in B\left(\Sigma_{0}\right)$. And there exist a (necessarily unique) "gate" $\phi_{\Sigma_{0}} \in B\left(\Sigma_{0}\right)$ for any map $\phi \in V(\Sigma)$, i.e., a map in $B\left(\Sigma_{0}\right)$ with $D(\phi, \psi)=D\left(\phi, \phi_{\Sigma_{0}}\right)+D\left(\phi_{\Sigma_{0}}, \psi\right)$ for all $\psi \in B\left(\Sigma_{0}\right)$, which is given by

$$
\phi_{\Sigma_{0}}(S):=\left\{\begin{array}{l}
\phi(S) \text { if } S \in \Sigma_{0}, \\
A\left(S \searrow \Sigma_{0}\right) \text { otherwise }
\end{array}\right.
$$

and must necessarily be contained in cut $(\Sigma)$ in case $\phi \notin B\left(\Sigma_{0}\right)$ holds. 
In particular, given any two distinct connected components $\Sigma_{0}, \Sigma_{1} \in$ $\pi_{0}(\Sigma)$ of the incompatibility graph of $\Sigma$, the map defined by

$$
\phi_{\Sigma_{0} \mid \Sigma_{1}}: \Sigma \rightarrow \mathcal{P}(X): S \mapsto\left\{\begin{array}{l}
A\left(S \searrow \Sigma_{1}\right) \text { if } S \in \Sigma_{0}, \\
A\left(S \searrow \Sigma_{0}\right) \text { otherwise, }
\end{array}\right.
$$

is the unique gate $\phi_{\Sigma_{0}} \in B\left(\Sigma_{0}\right)$ of all maps $\phi \in B\left(\Sigma_{1}\right)$. And any map in the intersection $B\left(\Sigma_{0}\right) \cap B\left(\Sigma_{1}\right)$ of the blocks $B\left(\Sigma_{0}\right)$ and $B\left(\Sigma_{1}\right)$, if there is any such map, must be a cut vertex of $\mathcal{B}(\Sigma)$. Furthermore, the following assertions all are equivalent:

$(B \cap B: 1)$ There exists some $\phi \in \operatorname{cut}(\Sigma)$ with $\phi \in B\left(\Sigma_{0}\right) \cap B\left(\Sigma_{1}\right)$.

$(B \cap B: 2) B\left(\Sigma_{0}\right) \cap B\left(\Sigma_{1}\right)$ is non-empty.

$(B \cap B: 3) B\left(\Sigma_{0}\right) \cap B\left(\Sigma_{1}\right)$ is a one-vertex set.

$(B \cap B: 4) A\left(S \searrow \Sigma_{0}\right)=A\left(S \searrow \Sigma_{1}\right)$ holds for all $S \in \Sigma-\left(\Sigma_{0} \cup \Sigma_{1}\right)$. I.e., there exists no split $S=\{A, B\} \in \Sigma-\left(\Sigma_{0} \cup \Sigma_{1}\right)$ with $A \cap A_{0}=\emptyset$ for some set $A_{0}$ in some split $S_{0} \in \Sigma_{0}$ and $B \cap B_{1}=\emptyset$ for some set $B_{1}$ in some split $S_{1} \in \Sigma_{1}$.

And in case all of these assertions hold, the map $\phi_{\Sigma_{0} \mid \Sigma_{1}} \in B\left(\Sigma_{0}\right)$ coincides with the correspondingly defined map $\phi_{\Sigma_{1} \mid \Sigma_{0}}$, and it is the unique map in $B\left(\Sigma_{0}\right) \cap B\left(\Sigma_{1}\right)$.

Finally, mapping each connected component $\Sigma_{0}$ of the incompatibility graph of $\Sigma$ onto the associated block $B\left(\Sigma_{0}\right)$ induces a canonical bijection

$$
\Psi=\Psi_{\Sigma}: \pi_{0}(\Sigma) \rightarrow B l(\Sigma): \Sigma_{0} \mapsto B\left(\Sigma_{0}\right)
$$

from the set $\pi_{0}(\Sigma)$ of connected components of $\Gamma(\Sigma)$ onto the set $B l(\Sigma)$ of all blocks of $\mathcal{B}(\Sigma)$.

Proof: To simplify the exposition of the proof, we will present it as a series of 12 observations: (i) It follows immediately from the definitions and Assertion (4.6) that $B\left(\Sigma_{0}\right)$ is a subset of $V(\Sigma)$. It is also obvious that $\Theta$ is an isometry as, by definition, the even stronger assertion

$$
\Delta(\widetilde{\phi}, \widetilde{\psi})=\Delta(\phi, \psi)
$$

apparently holds for all $\phi, \psi \in V\left(\Sigma_{0}\right)$. Hence, the subgraph induced by $\mathcal{B}(\Sigma)$ on $B\left(\Sigma_{0}\right)$ is an isometric subgraph of $\mathcal{B}(\Sigma)$.

(ii) We clearly have

$$
B\left(\Sigma_{0}\right)=\left\{\phi \in V(\Sigma): \forall_{S \in \Sigma-\Sigma_{0}} \phi(S)=A\left(S \searrow \Sigma_{0}\right)\right\}
$$

as $\phi(S)=A\left(S \searrow \Sigma_{0}\right)$ holds, by definition, for all $\phi \in B\left(\Sigma_{0}\right)$ and all $S \in \Sigma-\Sigma_{0}$. Conversely, given any map $\phi \in V(\Sigma)$ with $\phi(S)=A(S \searrow$ $\left.\Sigma_{0}\right)$ for all $S \in \Sigma-\Sigma_{0}$, we have $\psi:=\left.\phi\right|_{\Sigma_{0}} \in V\left(\Sigma_{0}\right)$ and $\widetilde{\psi}=\phi$.

(iii) Further, if $\phi\left(S^{\prime}\right)=A\left(S^{\prime} \searrow \Sigma_{0}\right)$ holds for all $S^{\prime} \in \Sigma-\Sigma_{0}$, $\phi\left(S^{\prime}\right) \cap A \neq \emptyset$ must hold for all $S^{\prime} \in \Sigma-\Sigma_{0}$ and subsets $A$ in any split $S \in \Sigma_{0}$. Thus, if $\phi=\widetilde{\psi}$ holds for some map $\psi \in V\left(\Sigma_{0}\right), S \in \Sigma^{(\phi)}$ must hold for any $S \in \Sigma_{0}$ for which $\phi(S)=\psi(S)$ is a minimal subset in $\psi\left[\Sigma_{0}\right]=\left\{\psi\left(S^{\prime}\right): S^{\prime} \in \Sigma_{0}\right\}$. So, we have indeed $B\left(\Sigma_{0}\right) \subseteq\{\phi \in V(\Sigma)$ : $\left.\Sigma^{(\phi)} \cap \Sigma_{0} \neq \emptyset\right\}$, as required. 
(iv) Conversely, given any $\phi \in V(\Sigma)$ with $\Sigma^{(\phi)} \cap \Sigma_{0} \neq \emptyset$, Assertion (4.5) implies that one must have $\phi\left(S^{\prime}\right)=A\left(S^{\prime} \searrow S\right)$ for any split $S \in \Sigma^{(\phi)}$ and any split $S^{\prime} \in \Sigma$ that is compatible with $S$. Hence, $\phi\left(S^{\prime}\right)=A\left(S^{\prime} \searrow \Sigma_{0}\right)$ must hold for any split $S^{\prime} \in \Sigma-\Sigma_{0}$. Thus, putting $\psi:=\left.\phi\right|_{\Sigma_{0}}$, we have $\psi \in V\left(\Sigma_{0}\right)$ as well as $\phi=\widetilde{\psi}$. So, $\phi \in B\left(\Sigma_{0}\right)$ holds. Thus, also $\left\{\phi \in V(\Sigma): \Sigma^{(\phi)} \cap \Sigma_{0} \neq \emptyset\right\} \subseteq B\left(\Sigma_{0}\right)$ and, therefore, also $\left\{\phi \in V(\Sigma): \Sigma^{(\phi)} \cap \Sigma_{0} \neq \emptyset\right\}=B\left(\Sigma_{0}\right)$ must hold, as claimed.

(v) Next, choosing any fixed map $\phi_{0} \in B\left(\Sigma_{0}\right)$, we have also $B\left(\Sigma_{0}\right)=$ $\left.\left\{\phi \in V(\Sigma): \Delta\left(\phi, \phi_{0}\right) \subseteq \Sigma_{0}\right)\right\}$ as

$$
\Delta\left(\phi, \phi_{0}\right) \subseteq \Sigma_{0} \Longleftrightarrow \phi(S)=\phi_{0}(S)=A\left(S \searrow \Sigma_{0}\right)
$$

holds for all $\phi_{0} \in B\left(\Sigma_{0}\right)$ and $\phi \in V(\Sigma)$.

(vi) It is also obvious that the map $\phi_{\Sigma_{0}}$ as defined above is indeed contained in $B\left(\Sigma_{0}\right)$ and that, given any map $\phi \in V(\Sigma), \phi_{\Sigma_{0}}$ is indeed the (necessarily unique) map in $B\left(\Sigma_{0}\right)$ with $D(\phi, \psi)=D\left(\phi, \phi_{\Sigma_{0}}\right)+$ $D\left(\phi_{\Sigma_{0}}, \psi\right)$ for all $\psi \in B\left(\Sigma_{0}\right)$ and, hence, the "gate" of $\phi$ in $B\left(\Sigma_{0}\right)$. And $\phi_{\Sigma_{0}} \in \operatorname{cut}(\Sigma)$ must hold in case $\phi \notin B\left(\Sigma_{0}\right)$ as it 'separates' $\phi$ from $B\left(\Sigma_{0}\right)$. I.e., there must be edges incident with $\phi_{\Sigma_{0}}$ whose types must be contained in distinct connected components of $\Gamma(\Sigma)$, those leading to $\phi$ and those leading to any map $\psi \in B\left(\Sigma_{0}\right)$ distinct from $\phi_{\Sigma_{0}}$ which must exist as the cardinality of $B\left(\Sigma_{0}\right)$ must be at least 2 for any connected component $\Sigma_{0}$ of $\Gamma(\Sigma)$.

(vii) Next, given any two distinct connected components $\Sigma_{0}, \Sigma_{1} \in$ $\pi_{0}(\Sigma)$, let $\psi$ denote the map $\psi:=\phi_{\Sigma_{0} \mid \Sigma_{1}}: \Sigma \rightarrow \mathcal{P}(X)$ that maps any split $S \in \Sigma_{0}$ onto $A\left(S \searrow \Sigma_{1}\right)$ and any other split $S \in \Sigma$ onto $A\left(S \searrow \Sigma_{0}\right)$. It is obvious that the restriction $\left.\psi\right|_{\Sigma_{0}}: S \mapsto A\left(S \searrow \Sigma_{1}\right)$ of $\psi$ to $\Sigma_{0}$ is contained in $V\left(\Sigma_{0}\right)$ in view of Assertion (4.6). And it is also obvious that $\psi$ coincides with the extension $\widetilde{\left.\psi\right|_{\Sigma_{0}}}$ of this restriction $\left.\psi\right|_{\Sigma_{0}}$. So, it is contained in $B\left(\Sigma_{0}\right)$.

It is also the gate $\phi_{\Sigma_{0}}$ in $B\left(\Sigma_{0}\right)$ of any map $\phi \in B\left(\Sigma_{1}\right)$ as, by definition, also $\phi_{\Sigma_{0}}(S)=\phi(S)=A\left(S \searrow \Sigma_{1}\right)=$ holds for all $S \in \Sigma_{0}$ and $\phi_{\Sigma_{0}}(S)=A\left(S \searrow \Sigma_{0}\right)$ holds for all $S \notin \Sigma_{0}$.

(viii) Furthermore, any map $\phi \in B\left(\Sigma_{0}\right) \cap B\left(\Sigma_{1}\right)$ must be a cut vertex of $\mathcal{B}(\Sigma)$ : Indeed, (7) implies that there must be edges incident with any such $\phi$ whose types are contained in $\Sigma_{0}$ as well as edges whose types are contained in $\Sigma_{1}$. Thus, the assertions $(B \cap B: 1)$ and $(B \cap B: 2)$ are indeed equivalent and follow from $(B \cap B: 3)$. Further, if $\phi \in$ $B\left(\Sigma_{0}\right) \cap B\left(\Sigma_{1}\right)$ holds, we must have $\phi(S)=A\left(S \searrow \Sigma_{0}\right)$ for all $S \in \Sigma-\Sigma_{0}$ and $\phi(S)=A\left(S \searrow \Sigma_{1}\right)$ for all $S \in \Sigma-\Sigma_{1}$. So, there can be only one such map, and $A\left(S \searrow \Sigma_{0}\right)=A\left(S \searrow \Sigma_{1}\right)$ must hold for all $S \in \Sigma-\left(\Sigma_{0} \cup \Sigma_{1}\right)$ in case such a map exists. So, " $(B \cap B: 2) \Longleftrightarrow(B \cap B: 3)$ " and " $(B \cap B: 2) \Rightarrow(B \cap B: 4) "$ holds.

(ix) And if $(B \cap B: 4)$ holds, i.e., if $A\left(S \searrow \Sigma_{0}\right)=A\left(S \searrow \Sigma_{1}\right)$ holds for all $S \in \Sigma-\left(\Sigma_{0} \cup \Sigma_{1}\right)$, there exists, of course, no split $S=\{A, B\} \in$ 
$\Sigma-\left(\Sigma_{0} \cup \Sigma_{1}\right)$ with $A \cap A_{0}=\emptyset$ for some set $A_{0}$ in some split $S_{0} \in \Sigma_{0}$ and $B \cap B_{1}=\emptyset$ for some set $B_{1}$ in some split $S_{1} \in \Sigma_{1}$ : Indeed, this would imply $A\left(S \searrow \Sigma_{0}\right)=B \neq A\left(S \searrow \Sigma_{1}\right)=A$.

Furthermore, the map $\psi=\phi_{\Sigma_{0} \mid \Sigma_{1}}$ coincides with the correspondingly defined map $\phi_{\Sigma_{1} \mid \Sigma_{0}}$. Indeed, Assertion $(B \cap B: 4)$ implies that both of these maps coincide: Both coincide with $A\left(S \searrow \Sigma_{0}\right)=A\left(S \searrow \Sigma_{1}\right)$ on all splits $S$ in $\Sigma-\left(\Sigma_{0} \cup \Sigma_{1}\right)$ while they both coincide with $A\left(S \searrow \Sigma_{1}\right)$ if $S \in \Sigma_{0}$ and with $A\left(S \searrow \Sigma_{9}\right)$ if $S \in \Sigma_{1}$. So, $\psi$ must the unique gate in $B\left(\Sigma_{0}\right)$ of every map in $B\left(\Sigma_{1}\right)$ and, simultaneously, the unique gate in $B\left(\Sigma_{1}\right)$ of every map in $B\left(\Sigma_{2}\right)$. Thus, $B\left(\Sigma_{1}\right) \cap B\left(\Sigma_{2}\right) \neq \emptyset$ must hold. I.e., $(B \cap B: 4)$ implies also $(B \cap B: 2)$. So, all of the assertions $(B \cap B: i) i=1,2,3,4$ must indeed be equivalent, as claimed.

(x) Furthermore, as $\mathcal{B}\left(\Sigma_{0}\right)$ is 2-connected (cf. Corollary 4.5), it follows from the definition of a block that $B\left(\Sigma_{0}\right)$ is a subset of some block $B \in B l(\Sigma)$. However, $B\left(\Sigma_{0}\right)$ must, in fact, coincide with that block $B$ : Indeed, $\phi_{0} \in B\left(\Sigma_{0}\right)$ and $\phi_{1} \in B$ implies that, by the definition of a block, $\Gamma_{\phi}\left(V^{(\phi)}, \phi_{0}\right)=\Gamma_{\phi}\left(V^{(\phi)}, \phi_{1}\right)$ must hold for all $\phi \in V(\Sigma)-\left\{\phi_{0}, \phi_{1}\right\}$. Thus, by Corollary 4.5, there must exist some connected component $\Sigma_{0}^{\prime}$ of the incompatibility graph $\Gamma(\Sigma)$ of $\Sigma$ with $\Delta\left(\phi_{0}, \phi_{1}\right) \subseteq \Sigma_{0}^{\prime}$. Moreover, by replacing $\phi_{0}$ by any map $\phi_{0}^{\prime} \in B\left(\Sigma_{0}\right)$ with $\emptyset \neq \Delta\left(\phi_{0}, \phi_{0}^{\prime}\right)$ if necessary, we may also assume that $\Delta\left(\phi_{0}, \phi_{1}\right) \cap \Sigma_{0} \neq \emptyset$ must hold and, therefore, $\Sigma_{0}=\Sigma_{0}^{\prime} \supseteq \Delta\left(\phi_{0}, \phi_{1}\right)$. This, in turn, implies that also $\phi_{1} \in B\left(\Sigma_{0}\right)$ and, therefore, also $B=B\left(\Sigma_{0}\right)$ must hold.

(xi) We clearly have $B\left(\Sigma_{0}\right) \neq B\left(\Sigma_{0}^{\prime}\right)$ for any two distinct connected components $\Sigma_{0}, \Sigma_{0}^{\prime}$ in $\pi_{0}(\Sigma)$, (as, e.g., $\Delta(\phi, \psi) \subseteq \Sigma_{0}$ and $\Delta\left(\phi^{\prime}, \psi^{\prime}\right) \subseteq \Sigma_{0}^{\prime}$ must hold for any $\phi, \psi \in B\left(\Sigma_{0}\right)$ and $\left.\phi^{\prime}, \psi^{\prime} \in B\left(\Sigma_{0}^{\prime}\right)\right)$. Thus, the map $\Psi$ is injective.

(xii) And finally, $\Psi$ is also surjective as there exists, for any block $B \in B l(\Sigma)$, some connected component $\Sigma_{0} \in \pi_{0}(\Sigma)$ of $\Gamma(\Sigma)$ with $B\left(\Sigma_{0}\right)=B$. Indeed, choose any edge $e:=\left\{\phi_{1}, \phi_{2}\right\}$ in $B$, let $S$ be the unique split in $\Delta\left(\phi_{1}, \phi_{2}\right)$, and let $\Sigma_{0}=\Gamma(\Sigma, S)$ be the connected component of $\Gamma(\Sigma)$ that contains the split $S \in \Sigma$. Then, $S \in \Sigma^{\left(\phi_{1}\right)} \cap \Sigma^{\left(\phi_{2}\right)}$ implies, in view of Assertion (4.5), that $\phi_{1}, \phi_{2} \in B\left(\Sigma_{0}\right)$ must hold. However, according to well-known properties of blocks, this can be true only if $B=B\left(\Sigma_{0}\right)$ holds. This completes the proof of the theorem.

\section{The $X$-Tree Associated With $\Sigma$}

Let us recall first that, given any connected simple graph $\Gamma=(V, E)$ with vertex set $V$ and edge set $E \subseteq\left(\begin{array}{l}V \\ 2\end{array}\right)$, the graph $\mathcal{T}(\Gamma)$ with vertex set $\mathcal{V}(\Gamma)$ the disjoint union $B l(\Gamma) \amalg V$ of the set $B l(\Gamma)$ of all blocks of $\Gamma$ and the set $V$ of all of its vertices, and edge set $\mathcal{E}(\Gamma)$ the set of all pairs $\{B, v\}$ with $B \in B l(\Gamma)$ and $v \in B$ is well-known to always be a tree (cf. [9, Proposition 3.1.2]). Clearly, the degree $\operatorname{deg}_{\mathcal{T}(\Gamma)}(v)$ of any vertex $v \in V$, considered as a vertex in $\mathcal{T}(\Gamma)$, coincides with the number of 
blocks that contain it, and the degree $\operatorname{deg}_{\mathcal{T}(\Gamma)}(B)$ of any block $B$ of $\Gamma$, considered as a vertex in $\mathcal{T}(\Gamma)$, coincides with the number of vertices it contains.

In consequence, continuing with the notation introduced so far, every collection $\Sigma$ of $X$-splits gives rise to a tree $\mathcal{T}(\Sigma):=\mathcal{T}(\mathcal{B}(\Sigma)$ ) with vertex set $\mathcal{V}(\Sigma):=B l(\Sigma) \amalg V(\Sigma)$, the disjoint union of $B l(\Sigma)$ and $V(\Sigma)$, and edge set the set $\mathcal{E}(\Sigma)$ of all pairs $\{B, \phi\}$ with $B \in B l(\Sigma)$ and $\phi \in B$. Moreover, the canonical labelling map $\phi_{\Sigma}: X \rightarrow V(\Sigma): x \mapsto \phi_{x}$ from $X$ into the $V(\Sigma)$ can also be viewed as a labelling map from $X$ into the vertex set $\mathcal{V}(\Sigma)$ of $\mathcal{T}(\Sigma)$.

Note further that the degree $\operatorname{deg}_{\mathcal{T}(\Sigma)}(B)$ of any block $B$ of $\mathcal{B}(\Sigma)$, considered as a vertex in $\mathcal{T}(\Sigma)$, coincides with the number of vertices it contains. In addition, the degree $\operatorname{deg}_{\mathcal{T}(\Sigma)}(\phi)$ of any vertex $\phi \in V(\Sigma)$ of $\mathcal{B}(\Sigma)$, considered as a vertex in $\mathcal{T}(\Sigma)$, coincides with the number of blocks that contain it and, hence, with the cardinality of $\pi_{\phi}\left(\Sigma^{(\phi)}\right)$, $\pi_{\phi}(\Sigma), \pi_{\phi}\left(X^{(\phi)}\right)$ as well as of $\pi_{\phi}\left(V^{(\phi)}\right)$. In particular, a vertex $\phi \in V(\Sigma)$ is of degree larger than 1 in $\mathcal{T}(\Sigma)$ if and only if it is a cut vertex of $\mathcal{B}(\Sigma)$.

In consequence, it is fairly obvious that we can also associate, to any system $\Sigma$ of $X$-splits, a "reduced" tree $T_{\Sigma}$ which is a "proper" $X$-tree: All one needs to do is (i) to delete all non-labeled vertices $\phi \in \mathcal{V}(\Sigma)$ with $\phi \in V(\Sigma)-\operatorname{cut}(\Sigma)$ and the pendant edges leading to them and (ii) to suppress all vertices of degree 2 , i.e., to replace each maximal sequence $u_{0}, u_{1}, \ldots, u_{k}$ of distinct non-labeled vertices of $\mathcal{T}(\Sigma)$ with $\left\{u_{i-1}, u_{i}\right\} \in \mathcal{E}(\Sigma)$ for all $i=1, \ldots, k$ and $\operatorname{deg}_{T_{\Sigma}}\left(u_{i}\right)=2$ for all $i=1, \ldots, k-1$ by just one edge $\left\{u_{0}, u_{k}\right\}$ while simultaneously deleting all the vertices $u_{1}, u_{2}, \ldots, u_{k-1}$ in between $u_{0}$ and $u_{k}$ and the edges incident with them, see also Figure 2.

Thus, a block $B$ in $B l(\Sigma)$ of the form $B=B\left(\Sigma_{0}\right)$ for some $\Sigma_{0} \in$ $\pi_{0}(\Sigma)$ will be suppressed if and only if $\Sigma_{0}$ consists of a single split, only. Otherwise, its degree $\operatorname{deg}_{T_{\Sigma}}(B)$ in the "reduced" tree $T_{\Sigma}$ coincides with the number of equivalence classes of the equivalence relation $\sim_{\Sigma_{0}}$ defined on $X$ by

$$
x \sim_{\Sigma_{0}} y \Longleftrightarrow \forall_{S \in \Sigma_{0}} S(x)=S(y) .
$$

In particular, the labelling map $\phi_{\Sigma}$ sets up a bijection from $X$ onto the set of leaves of $\mathcal{T}(\Sigma)$ if and only if $\operatorname{cut}(\Sigma) \cap\left\{\phi_{x}: x \in X\right\}=\emptyset$ and the equivalence relation $\sim_{\Sigma}$ is the identity relation on $X$, i.e., $\bigcap_{S \in \Sigma} S(x)=$ $\{x\}$ holds for all $x \in X$.

Altogether, this implies

Theorem 6.1. Suppose as above that $X$ is a finite set of cardinality at least 2 , and that $\Sigma$ is a collection of X-splits. Then, the following holds:

(i) The tree $\mathcal{T}(\Sigma)=\mathcal{T}(\mathcal{B}(\Sigma)$ ) canonically associated with the graph $\mathcal{B}(\Sigma)$ is isomorphic to the graph with vertex set the disjoint union of 


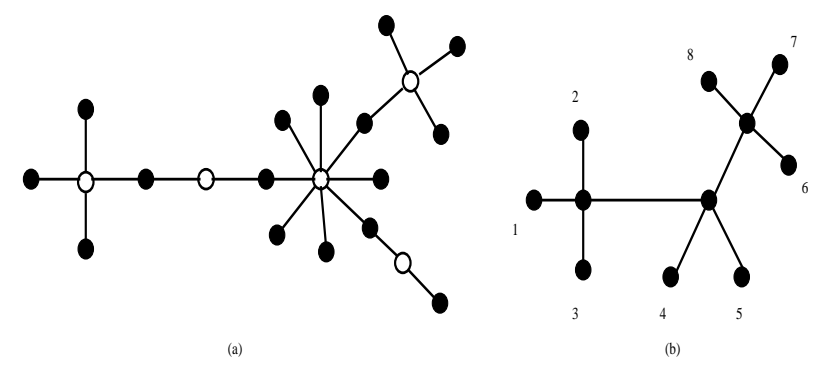

Figure 2. (a) The graph $\mathcal{T}\left(\Sigma_{8}\right)$ corresponding to the Buneman graph $\mathcal{B}\left(\Sigma_{8}\right)$ in Figure 1, where white vertices correspond to elements in the set $B l\left(\Sigma_{8}\right)$ and black vertices to those in $V\left(\Sigma_{8}\right)$. (b) The $X$-tree $T_{\Sigma_{8}}$ obtained from the tree $\mathcal{T}\left(\Sigma_{8}\right)$ by deleting all non-labeled vertices $\phi \in \mathcal{V}(\Sigma)$ with $\phi \in V(\Sigma)-\operatorname{cut}(\Sigma)$ and the pendant edges leading to them and suppressing all vertices of degree 2 in $\mathcal{T}\left(\Sigma_{8}\right)$. Note that the split system $\left\{S_{1}, S_{2}, S_{3}, S_{123}, S_{4}, S_{5}, S_{678}, S_{6}, S_{7}, S_{8}\right\}$ gives rise to the same $X$-tree.

$\pi_{0}(\Sigma)$ and $V(\Sigma)$, and edge set

$$
\left\{\left\{\Sigma_{0}, \phi\right\}: \Sigma_{0} \in \pi_{0}(\Sigma), \phi \in V(\Sigma), \Sigma^{(\phi)} \cap \Sigma_{0} \neq \emptyset\right\} .
$$

(ii) The graph obtained from the tree $\mathcal{T}(\Sigma)$ by deleting all non-labeled vertices $\phi \in V(\Sigma)-\operatorname{cut}(\Sigma)$ and the pendant edges leading to them as well as "suppressing" all non-labeled vertices of degree 2 in $\mathcal{T}(\Sigma)$ $i s$, together with the induced labelling map from $X$ into its vertex set, an $X$-tree that we denote by $T_{\Sigma}$. It is canonically associated with $\Sigma$ and coincides with that $X$-tree that, according to Peter Buneman, is associated with $\Sigma$ in case $\Sigma$ is compatible.

(iii) Furthermore, given any distinct blocks $B_{1}, B_{2} \in B l(\Sigma)$ for which a cut vertex $\phi \in B_{1} \cap B_{2}$ exists, one has

$$
\max \left(\operatorname{deg}_{\mathcal{T}(\Sigma)}\left(B_{1}\right), \operatorname{deg}_{\mathcal{T}(\Sigma)}\left(B_{2}\right), \operatorname{deg}_{\mathcal{T}(\Sigma)}(\phi)\right) \geq 3,
$$

that is, at least one of the three vertices $B_{1}, B_{2}$, and $\phi$ of $\mathcal{T}(\Sigma)$ must, for every such triple $B_{1}, B_{2}$, and $\phi$, also be a vertex of the $X$-tree $T_{\Sigma}$ derived from $\mathcal{T}(\Sigma)$.

Proof: The first two assertions follow immediately from our previous observations. To establish (iii), note that since $B_{1} \neq B_{2}$ we have $\operatorname{deg}_{\mathcal{T}(\Sigma)}\left(B_{1}\right), \operatorname{deg}_{\mathcal{T}(\Sigma)}\left(B_{2}\right) \geq 2 . \operatorname{But}_{\operatorname{deg}_{\mathcal{T}(\Sigma)}}\left(B_{1}\right), \operatorname{deg}_{\mathcal{T}(\Sigma)}\left(B_{2}\right)=2$ would imply that there exist two distinct vertices $\psi_{1}, \psi_{2} \in V(\Sigma)-\{\phi\}$ with $B_{1}=\left\{\psi_{1}, \phi\right\}$ and $B_{2}=\left\{\psi_{2}, \phi\right\}$. Let $S_{1}, S_{2} \in \Sigma$ denote the splits for which $\Delta\left(\psi_{1}, \phi\right)=\left\{S_{1}\right\}$ and $\Delta\left(\psi_{2}, \phi\right)=\left\{S_{2}\right\}$ both hold. Then $A_{1} \cup A_{2}=X$ must hold for $A_{1}:=\phi\left(S_{1}\right)$ and $A_{2}:=\phi\left(S_{2}\right)$. This, in turn, implies that $A_{1} \cap A_{2} \neq \emptyset$ must hold as well as $S_{1}, S_{2} \notin \Delta\left(\phi, \phi_{x}\right)$ for every $x \in A_{1} \cap A_{2}$. Hence, in view of Lemma 4.2 and Theorem 5.1. $\Gamma_{\psi}\left(\Sigma^{(\phi)}\right)$ contains at least three connected components: $\left\{S_{1}\right\},\left\{S_{2}\right\}$, and 
the connected component containing $\Delta_{\min }\left(\phi \mid \phi_{x}\right)$ for some $x \in A_{1} \cap A_{2}$.

As mentioned already above, we will explore these matters in more detail in [15]. We will consider in particular the graph theoretical invariant, defined according to [14], of the Buneman complex $\mathbf{B}(\Sigma)$ associated to $\Sigma$ as defined in [12]. And we will show that $\mathcal{B}(\Sigma)$ as well as $\mathbf{B}(\Sigma)$ can be described as a push-out in terms of the data involved in the construction of $T_{\Sigma}$ suggesting efficient algorithms for their computation (see also [17, 18]).

In yet another paper, we will discuss what can be done in case the incompatibility graph $\Gamma(\Sigma)$ is connected and $\mathcal{B}(\Sigma)$ is, hence, 2connected. In particular, by directing attention towards cut faces rather than merely cut vertices of $\mathcal{B}(\Sigma)$, we show that it may be possible even in this case to extract valuable phylogenetic information from a split system $\Sigma$.

Acknowledgment: The authors thank the Engineering and Physical Sciences Research Council (EPSRC) for its support [Grant EP/D068800/1], and also Andreas Spillner for helpful discussions. K. T. Huber and J.H. Koolen thank the Royal Society for its support through their International Joint Projects scheme. J. H. Koolen was also partially supported by the Priority Research Centers Program through the National Research Foundation of Korea (NRF) funded by the Ministry of Education, Science and Technology (Grant 2009-0094069). A. Dress thanks the Chinese Academy for Sciences, the Max-Planck-Gesellschaft, and the German BMBF for their support, as well as the Warwick Institute for Advanced Study where, during two wonderful weeks, the basic outline of this paper was conceived. Finally, we also thank an anonymous referee for his/her helpful comments on the first version of this paper.

\section{REFERENCES}

[1] J. Barthélemy, From copair hypergraphs to median graphs with latent vertices, Disc. Math., 76 (1989), pp. 9-28.

[2] J. Barthélemy, A. Guenoche, Trees and Proximity Representations, John Wiley \& Sons, Chichester New York Brisbane Toronto Singapore, 1991.

[3] H. -J.Bandelt, Generating median graphs from Boolean matrices, in $L_{1}$ statistical analysis and related methods, Y. Dodge, editor, North-Holland, 1992, pp. 305-309.

[4] H. J. Bandelt, A. Dür, Translating DNA data tables into quasi-median networks for parsimony analysis and error detection, Mol. Phylog. and Evol., 42 (2007), pp. 256-271.

[5] H. -J. Bandelt, P. Forster, B. C. Sykes, M. B. Richards, Mitochondrial portraits of human populations using median networks, Genetics, 141 (1995), pp. 743753.

[6] H. J. Bandelt, P. Forster, A. Röhl, Median-joining networks for inferring intraspecific phylogenies, Mol. Bio. and Evol., 16(1) (1999), pp. 37-48.

[7] B. Brešar, S. Klavžar, Maximal proper subgraphs of median graphs, Disc. Math., 307 (2007), pp. 1389-1394. 
[8] P. Buneman, The recovery of trees from measures of dissimilarity, in Mathematics in the Archaeological and Historical Sciences, F. Hodson et al. editors, Edinburgh University Press, 1971, pp. 387-395.

[9] R. Diestel, Graph Theory, Third Edition, Springer-Verlag, Heidelberg, 2005.

[10] A. Dress, S. Grünewald, LI Jun, The H1N1 Flu: Its evolution in pictures (2009) http://www.picb.ac.cn/picb-dynamic/Desktop/news/shownews.jsp?ID=538

[11] A. Dress, M. Hendy, K. Huber, V. Moulton, On the number of vertices and edges in the Buneman Graph, Ann. of Comb., 1 (1997), pp. 329-337.

[12] A. Dress, K. T. Huber, V. Moulton, Some variations on a theme by Buneman, Ann. of Comb., 1 (1997), pp. 339-352.

[13] A. Dress, K. T. Huber, J. Koolen, V. Moulton, Cut points in metric spaces, App. Math. Letters, 21(6) (2008), pp. 545-548.

[14] A. Dress, K. T. Huber, J. Koolen, V. Moulton, A graph-theoretical invariant of topological spaces, App. Math. Letters, 22(2) (2009) 159-162.

[15] A. Dress, K. T. Huber, J. Koolen, V. Moulton, A canonical decomposition of the Buneman complex, in preparation.

[16] A. Dress, R. Scharlau, Gated sets in metric spaces, Aequ. Math., 34 (1987), pp. 112-120.

[17] D. Gusfield, V. Bansal, A fundamental decomposition theory for phylogenetic networks and incompatible characters, in Lecture Notes in Computer Science 3500, T. Speed, H. Huang, editors, Springer, 2005, pp. 217-232.

[18] D. Gusfield, V.Bansal, V.Bafna, Y.Song, A decomposition theory for phylogenetic networks and incompatible characters, J. Comp. Bio., 14(10) (2007), pp. 1247-1272.

[19] D. H. Huson, T. Kloepper, Beyond galled trees: Decomposition and computation of galled networks, in Lecture Notes in Computer Science 4453, S. Miyano et al., editors, Springer, 2007, pp. 211-225.

[20] S. Klavžar, H. M. Mulder, Median graphs: characterizations, location theory and related structures, J. Combin. Math. Combin. Comput., 30 (1999), pp. 103-127.

[21] C. Semple, M. Steel, Phylogenetics, Oxford University Press, 2003.

[22] S. Sridhar, F. Lam, G. Blelloch, R. Ravi, R. Schwartz, Efficiently finding the most parsimonious phylogenetic tree via linear programming, in Lecture Notes in Computer Science 4463, I. Mandoiu, A. Zelikovsky, editors, Springer, 2007, pp. 37-48.

Andreas W. M. Dress, Department of Combinatorics and Geometry, CAS-MPG Partner Institute and Key Lab for Computational Biology, Shanghai Institutes for Biological Sciences, Chinese Academy of ScIences, Shanghai, China, and Max Planck Institute for Mathematics in the Sciences, D-04103 Leipzig, Germany, Email: Andreas@picb.aC.CN, Katharina T. Huber, School of Computing Sciences, University of East AngLia, Norwich, NR4 7TJ, UK, EMAIL: Katharina.Huber@CMP.UEa.AC.UK, Jacobus Koolen, Pohang Mathematics Institute and Department of Mathematics, POSteCH, Hyoja-dong, Namgu Pohang, 790-784 South Korea, EMail: KoOlen@postech.AC.KR, Vincent Moulton, School of Computing Sciences, University of East Anglia, Norwich, NR4 7TJ, UK, EMAIL: VINCENT.MOULTON@CMP.UEA.AC.UK 